\title{
A Study on the Statistical Properties of the Prime Numbers Using the Classical and Superstatistical Random Matrix Theories
}

\author{
M. Abdel-Mageed $\mathbb{D}^{1,2}$ Ahmed Salim $\mathbb{D}^{2,3}$ Walid Osamy $\mathbb{D}^{4,5}$ and Ahmed M. Khedr $\mathbb{D}^{2,6}$ \\ ${ }^{1}$ Department of Physics, Unaizah College of Science and Arts, Qassim University, Qassim, Saudi Arabia \\ ${ }^{2}$ Faculty of Science, Zagazig University, Zagazig, Egypt \\ ${ }^{3}$ Department of Computer Science, College of Science and Arts, Al-Mithnab, Qassim University, P.O. Box 931, Buridah, \\ 51931 Al-Mithnab, Saudi Arabia \\ ${ }^{4}$ Department of Applied Natural Science, College of Community, Qassim University, Unaizah, Saudi Arabia \\ ${ }^{5}$ Computer Science Department, Faculty of Computers and Artificial Intelligence, Benha University, Benha, Egypt \\ ${ }^{6}$ Computer Science Department, University of Sharjah, Sharjah 27272, UAE
}

Correspondence should be addressed to M. Abdel-Mageed; m.abdulasim@qu.edu.sa and Walid Osamy; w.elsherif@qu.edu.sa

Received 18 March 2021; Revised 10 August 2021; Accepted 13 August 2021; Published 22 September 2021

Academic Editor: Giorgio Kaniadakis

Copyright (c) $2021 \mathrm{M}$. Abdel-Mageed et al. This is an open access article distributed under the Creative Commons Attribution License, which permits unrestricted use, distribution, and reproduction in any medium, provided the original work is properly cited.

\begin{abstract}
The prime numbers have attracted mathematicians and other researchers to study their interesting qualitative properties as it opens the door to some interesting questions to be answered. In this paper, the Random Matrix Theory (RMT) within superstatistics and the method of the Nearest Neighbor Spacing Distribution (NNSD) are used to investigate the statistical proprieties of the spacings between adjacent prime numbers. We used the inverse $\chi^{2}$ distribution and the Brody distribution for investigating the regular-chaos mixed systems. The distributions are made up of sequences of prime numbers from one hundred to three hundred and fifty million prime numbers. The prime numbers are treated as eigenvalues of a quantum physical system. We found that the system of prime numbers may be considered regular-chaos mixed system and it becomes more regular as the value of the prime numbers largely increases with periodic behavior at logarithmic scale.
\end{abstract}

\section{Introduction}

Prime numbers are usually considered a mathematical tool for investigating mathematical and physical ideas. Prime numbers play an important role in number theory and cryptography. Because of this unique nature of prime numbers, they are mainly used in many Wireless Sensor Networks and Internet of Things applications such as security [1-7]. The distribution of prime numbers is considered to be directly related to the statistical distribution of the nontrivial zeros of the Riemann Zeta function that closely resembles the energy levels of atomic nuclei [8-11]. Nuclear spectra and the Zeta function zeros are therefore correlated through their statistical distribution since both spacings between the energy levels of a heavy atomic nucleus and the zeros of the Riemann Zeta function behave like spacings between eigenvalues of a random matrix $[9,10]$. The prime-number sequence, viewed as the spectrum of eigenvalues of random matrices, is found to be quasichaotic. Although chaotic, the distribution of prime numbers has a deterministic chaotic behavior, with symmetries and harmonics, that follows the classical path to chaos, i.e., from periodic to quasiperiodic to chaotic [12]. Over the last three decades, it has appeared in various fields of science including quantum chaos [13, 14], cryptology [15], and biology [16, 17].

One of the most attractive features of prime numbers is their distribution among the natural numbers. On a small scale, the distribution of prime numbers appears random; however, when considered on a large scale, it appears to have a pattern that is not fully understood. If we consider the frequency distribution of prime numbers up to a number, say $n$, even though it is difficult to detect a pattern on a small scale, the graph appears to have a much smoother curve as the value of $n$ becomes larger [18]. However, the unique 
pattern and properties of prime numbers make it challenging to take a clear decision about the nature of the spacings between prime numbers at very large values. At the same time, it is of great importance to study and analyze the nature of the spacings between the prime numbers at very large values because of their mysterious characteristics and advantages for applications in various routines, for example, in cryptography-related fields and other applications. Moreover, the search for a deterministic formula that can quickly generate large numbers that are guaranteed to be prime is still being explored. The research in finding answers to the interesting issues surrounding the prime numbers and their properties is still ongoing.

The problem of the prime numbers sequence being regular or chaotic is one of the axial problems nowadays. On a small scale, the distribution of prime numbers appears random; however, when considered on a large scale, it appears to have a pattern that is not fully understood. This interplay between regular and chaotic behaviors has motivated researchers for a detailed study in this area that could possibly be signatures of more fundamental mathematical properties and features [19]. The statistical properties of prime numbers are one way of investigating their behavior and characteristics. In general, several attempts to investigate the use of level-spacing distributions of quantum systems belonging to different classes related to the symmetry properties of the Hamiltonian, such as the Poisson distribution (for systems with underlying regular classical dynamics), Wigner-Dyson distribution (Hamiltonians invariant under time reversal), and Gaussian Unitary Ensemble (variant under time reversal), were conducted in analyzing the spacing distribution between adjacent prime numbers $[20,21]$. Even though there exist several reviews on this topic and several authors have investigated the statistical properties of the spacings between adjacent prime numbers [21-32], there still remains some confusion regarding the proper statistics of the spacings between consecutive prime numbers.

In this paper, we perform a new study on the statistical properties of prime numbers using the superstatistical Random Matrix Theory (RMT) [33-36]. The superstatistical theory was first introduced by Beck and Cohen to describe the deviation of a thermodynamic system from equilibrium $[37,38]$, and we are inspired by the success of the superstatistical RMT in describing the transition of mixed system's behavior between regular and chaotic [33-36, 39]. In this paper, we use the RMT and the method of the Nearest Neighbor Spacing Distribution (NNSD) to investigate the statistical properties of the spacings between adjacent prime numbers, where the prime numbers are treated as energy levels of physical quantum system [21, 28]. For analysis, we have used the inverse $\chi^{2}$ distribution [33-36, 39] and the Brody distribution which is a popular and powerful tool for investigating the regular-chaos mixed systems [40-44]. We conducted analysis on every million prime numbers separately, up to three hundred and fifty million prime numbers. Next, the distribution was made into sequences of first $N$ prime numbers from one hundred to three hundred and fifty million prime numbers.
The remainder of the paper is organized as follows: Section 2 gives the related research, and Section 3 discusses the background details on the level spacing statistics and RMT. The proposed study is provided in Section 4. The results and discussion are provided in Section 5. Section 6 concludes the paper.

\section{Related Research}

The topic of distribution of prime numbers, or more precisely, the spacing distribution between consecutive prime numbers, has attracted much attention recently. Moreover, the mysterious distribution behavior of prime numbers and their unexpected role in multiple contexts such as cryptography, quantum chaos, and biology have appealed for further investigation into their features $[13-17,45]$. This has made prime numbers into a prime object of interest to mathematicians and other researchers. Even after centuries of research, there still remain open challenges to be solved to reveal the exact details on the distribution and spacing between prime numbers.

In recent years, different methods have been applied to study prime numbers and their distribution. Some problems related to the gaps between prime numbers are well known, for example, Legendreâ's conjecture (which states that there is a prime number between $n^{2}$ and $(n+1)^{2}$ for every positive integer $n$ ) and the famous twin prime number conjecture [46].

While the multifractality of prime numbers was studied in [47], some appropriately defined Lyapunov exponents were calculated numerically for studying the distribution of prime numbers in [25], where Gamba et al. studied the difference between the prime counting function $(\pi(x))$ and its analytical approximation $(R(x))$. They studied 63950 prime numbers through $8 \times 10^{5}$ natural numbers and claimed the chaotic behavior of the prime numbers [26]. In [27], the author studied the first unfolded $10^{4}$ prime numbers and the prime numbers in the range between $10^{12}$ and $10^{12}+$ $10^{4}$ without unfolding; while the latter follow the Poissonian behavior, the first $10^{4}$ prime numbers did not match very well and concluded the Poissonian behavior for the first $N$ prime numbers as it approaches infinity.

In [28], the authors studied the prime numbers in the first million primes, increasing the steps by an order of magnitude starting from $10^{2}$ to $10^{6}$, and then studied the million after $10^{7}, 10^{8}, \ldots, 10^{12}$ th prime numbers. They also studied the variance for the same sequence of prime numbers and concluded the regularity and the Poissonian behavior of the prime numbers as the size of the prime numbers is largely increased. In [29], Liboff and Wong studied the NNSD for three sequences of unfolded first $N$ prime numbers at $5 \times 10^{4}, 10^{6}$, and $10^{7}$ prime numbers. They found that the sequences had a level repulsion with nearly chaotic behavior and a rough fit to Wigner distribution.

In [21], Wolf studied the NNSD for the first $N$ sequence of prime numbers up to very large prime numbers. The work studied 762939111 prime numbers as one sequence with three different bin sizes (see Figure 4 in Ref. [21]). The prime 
numbers are regarded as energy levels and physical methods used to study spectra of quantum systems were applied to the description of distribution of prime numbers. A presentation of heuristic arguments and computer results to support the hypothesis, namely, that the statistics of gaps between consecutive prime numbers follow the Poisson behavior after appropriate rescaling, is also provided. The scaling transformation removes the oscillations in the NNSD of prime numbers. The author also studied the spectral rigidity for different sets of prime numbers and derived the same conclusion.

In [12], the author showed that fractal theory can be utilized to analyze the distribution of prime numbers. Based on the similarities between the distribution of powers of prime numbers for each natural number and generalized Cantor's sets, some symmetric properties were analyzed and used to propose a set of discrete dynamics for visualizing the recursive and symmetric properties of prime numbers. This leads to the derivation of an approximation of a fractal version of the distribution function of prime numbers. Then, using this discrete dynamics set, they proposed a prime number's sieve algorithm which is analogous to the growth of a fractal. Their findings suggest that the fractal and chaotic behavior on the distribution of prime numbers emerges from the symmetry break due to the exponential growth of the length of the symmetries with respect to the domain of applicability of each discrete dynamics. Hence, they derived a conclusion that the prime number distribution exhibits deterministic chaotic behavior with symmetries and harmonics.

In [48], the authors studied and analyzed the large spacing between adjacent prime numbers. Their study gives the relation of the size of the largest gap between consecutive prime numbers less than $X$ on a function $f(X)$ that tends to infinity with $X$. Their work relies on the research that claims the existence and distribution of long arithmetic progressions consisting entirely of prime numbers, and their proof combines the existing arguments with a random construction covering a set of prime numbers by arithmetic progressions as well.

In [19], a simple representation of prime numbers in $2 \mathrm{D}$ is introduced that may lead to important avenues in the field of research on prime numbers. The proposed numerical representation yielded an interesting visual structure that depicts an oscillating plot, which ascends and descends according to the distribution of prime numbers. As the structure resembles a ladder, it was referred to as Jacob's ladder $(J(n))$. Even though the zeroes in the structure grow in an unpredictable manner, the gaps between them exhibit a remarkable and fascinating property which is a clear exponential decay in the frequency of gaps versus the gap size. The smaller the gaps, the more frequently they appear. This important result represents an unexpected correlation between the apparently chaotic sequence, the zeroes in $J(n)$, and the prime number distribution. Additionally, the sequence of zeroes, despite being nonconsecutive numbers, contains a number of prime numbers approximately equal to $n / \log n$, where $n$ refers to the number of terms in the sequence.

In [49], the authors aimed to derive heuristic formulas to predict the growth trend of the maximal spacing between adjacent prime numbers. They performed an extensive study on the maximal gaps between prime $k$-tuples in residue classes $(\bmod q)$. Their findings suggest that almost all the maximal gaps satisfy a generalized strong form of Cramer's conjecture.

In most of the previous studies, the authors took only small or separate regions of the prime numbers stream, except in [21], where the author studied a single very large sequence. However, no clear decision has been made regarding the nature of the spacings between prime numbers through the first $N$ sequences up to humongous large prime values. In this work, we study the spacing distribution between adjacent prime numbers using NNSD for a continuous stream of prime numbers up to three hundred and fifty million prime values, where the prime numbers were treated as eigenvalues of a quantum physical system of a simple onedimensional Hamiltonian [21, 27-29]. We applied the methods of RMT within superstatistics [33-37, 39, 50, 51] and Brody distribution [40] in this study.

\section{Background: Level Spacing Statistics and RMT}

Integrable and chaotic physical systems are very rare in nature. Most of the real physical systems are mixed, and the statistical properties of many quantum physical systems can be modeled by random matrices. Such a system is generally a many-particle system whose interaction is so complex that the Hamiltonian representing the system should behave like a large random matrix. This can mean that the Hamiltonian of such systems is mixed where it contains parts of regular and chaotic motion dynamics together. Furthermore, simple one-particle quantum systems may also exhibit random matrix statistics if the classical limit of the system is chaotic. A major aspect of quantum chaos theory is that distributions of properly normalized distances between neighboring energy eigenvalues for a quantum system can behave as a good indicator of the system properties and behavior [20,33-36].

Usually, the eigenvalues of quantum systems whose classical counterparts are regular have the Poisson level spacing distributions, whereas the eigenvalues of quantum systems with chaotic classical counterparts have random matrix level spacing distributions [52-54]. In addition, a classical system that undergoes a transition from regular motion to chaos as some parameter is varied should have a quantum counterpart whose eigenvalues undergo a transition from the Poisson to random matrix statistics for the same variation of the parameter. This gives the indication that Random Matrix Theory (RMT) can provide a successful model for quantum systems, whose classical counterpart has chaotic dynamics $[20,55,56]$.

The uncorrelatedness property of random numbers can be utilized to determine the distribution of gaps between the nearest neighbors in an ordered random number sequence. Moreover, in regular or nearly ordered systems, mixing of quantum states belonging to adjacent levels can be ignored and the energy levels are uncorrelated $[33,56-$ 58]. The sequence of prime numbers being statistically 
similar to a sequence of random numbers, and the imaginary parts of the nontrivial zeros of the Riemann Zeta function (numbers that are intimately tied to the detailed distribution of prime numbers) have statistical properties that fit the predictions of RMT [10,47]. Consider a numberNin the sequence; the probability $p_{p}(s) \cdot d s$ that the next number in the sequence lies between $(N+s)$ and $(N+s+d s)$ is proportional to the probability that there is no level between $N$ and $(N+s)$. This indicates that the Nearest Neighbor Spacing Distribution for random numbers is the Poisson distribution. More specifically, the distances between energy eigenvalues for a generic integrable system are distributed as for points randomly thrown on a line, which can be expressed by the Poisson distribution. That means, such a system can be described by the Poisson distribution given as follows:

$$
p_{p}(s)=e^{(-s)}
$$

where $p_{p}(s)$ has been properly normalized so that the mean spacing is one. Note that the Poisson distribution has the peak value at $s=0$, indicating that small gaps are very likely in a sequence of random numbers. This tendency of random numbers to clump together is referred to as level clustering $[56,59]$. At the same time, the level spacings of a chaotic system are expected to obey the same statistics as the eigenvalues of an ensemble of real symmetric random matrices with independent identically distributed entries [20, 55, 56]. Wigner recognized the importance of eigenvalue statistics when he studied the distribution of spacings between energy levels of highly excited nuclei, where he found that the nuclear energy levels were statistically similar to the eigenvalues of a random matrix, and their behavior mimics that of a random matrix. The experiments shown are found to fit the predictions of RMT [27]. Moreover, the NNSD statistics of mixed quantum systems are likely to execute a Poisson-to-Wigner transition as the underlying classical dynamics monotonically change from being completely integrable to being completely chaotic.

The chaotic phase of a system can therefore be described using the Wigner-Dyson distribution given by

$$
p_{w}(s)=\frac{\pi}{2} s e^{\left(-(\pi / 4) s^{2}\right)}
$$

Numerous interpolation formulas describing the intermediate situations between the complete integrability and chaos have been considered in $[33-36,39,40,55,56,60]$. On the derivation of a formula for mixed systems by generalizing RMT, Abul-Magd derived a formula which was carried out using the superstatistics to RMT $[33-36,39]$. He suggested using the idea of superstatistics that the mixed system is constructed from many microscopic cells that are momentarily in a chaotic phase. Every cell is sufficiently large to follow the essential statistics of RMT but with different distribution parameter $\eta$ related to it, due to a probability density $f(\eta)$.
Hence, the Random-Matrix ensemble within superstatistics that represents the mixed system is a combination of Gaussian ensembles [33-37, 39, 51, 61, 62]. Then, by applying the method of the inverse $\chi^{2}$ distribution of the superstatistical random matrix, the formula for the NNSD can be obtained:

$$
p_{\text {invchi }}{ }^{2}(v, s)=\frac{2 \eta_{0} s}{\Gamma(v / 2)}\left(\sqrt{\eta_{0} v s^{2}} / 2\right)^{v / 2} K_{v / 2}\left(\sqrt{\eta_{0} v s^{2}}\right),
$$

where $K_{m}(x)$ is the modified Bessel function [63] and $\eta_{0}$ is determined by the requirement that the mean level spacing $\langle s\rangle$ must equals unity:

$$
\eta_{0}=\frac{4 \pi}{v^{3}}\left[\frac{\Gamma((v+3) / 2)}{\Gamma(v / 2)}\right]^{2}
$$

Therefore, the assumption that the inverse square of the variance of matrix elements as an inverse $\chi^{2}$ variable allows one to model the regular-chaos mixed systems is applied [33-36, 39]. The distribution tends to the Poisson distribution as $v \longrightarrow-1$ and to the Wigner-Dyson distribution if $v \longrightarrow \infty$. In other words, as the values of $v$ increase from -1 to $\infty$, the chaoticity of the system increases. It is interesting to note that as $v \longrightarrow 1$, it yields the semi-Poisson distribution which can describe the spectra of pseudointegrable systems [64] (a more detailed discussion about Equation (3) can be found in Ref. [39]).

\section{Proposed Study}

The statistical properties of number sequences have helped to elucidate some interesting challenges in number theory, especially in the study of prime numbers [27, 33-36], motivated by the success of the superstatistical RMT in describing the transition of mixed system's behavior between regular and chaotic $[36,39]$. We propose a new study on the statistical properties of the prime numbers using the superstatistical RMT; we use RMT within superstatistics and apply the method of NNSD to investigate the statistical properties of the spacing between adjacent prime numbers $[20,22,33-38,51,55,56]$. The prime numbers are treated as energy levels of physical quantum systems [21, 28]. For analysis, we have used the inverse $\chi^{2}$ distribution [33-36, 39] and Brody distribution [40-44].

One of the reasons for using the superstatistical RMT in describing the mixed systems can be found in [36]; the authors considered two billiards with mushroom-shaped boundaries as representatives of systems with mixed regular-chaotic dynamics and three with the shape of Limacon billiards; one is chaotic dynamics and two are mixed dynamics. In all cases, the experimental data are found in better agreement with inverse- $\chi^{2}$ superstatistics than all the other considered distributions. Moreover, the inverse- $\chi^{2}$ distribution is employed in describing the symmetry breaking of acoustic resonances in quartz blocks which agrees well with experimental spectra of acoustic resonances than other distributions such as log-normal and $\chi^{2}$ distributions [39]. 


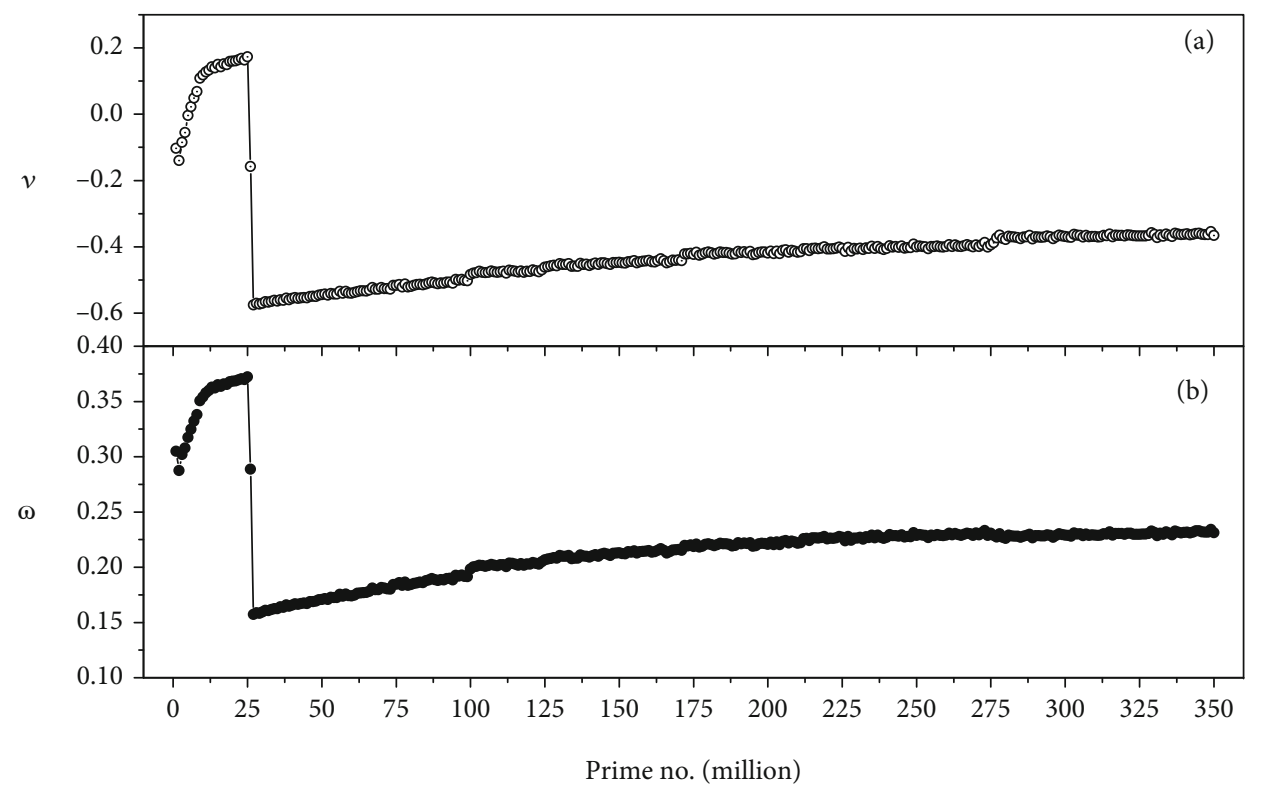

Figure 1: (a) The relation between the prime number of every million separately and the inverse $\chi^{2}$ and parameter $v$ from one million up to 350 million primes for every million separately. (b) The relation between the prime number and Brody parameter $\omega$ from one million up to 350 million primes for every million separately.

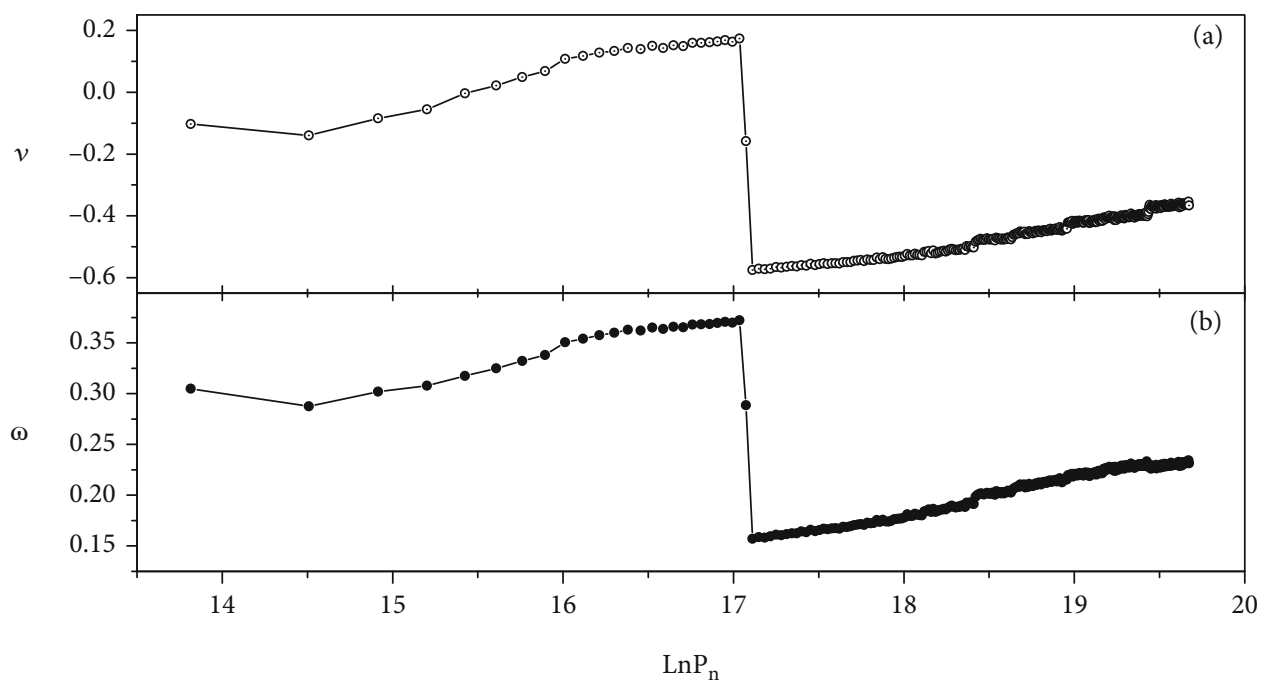

FIGURE 2: (a) The relation between the natural logarithm of the prime numbers and the inverse $\chi^{2}$ parameter $v$ from one million up to 350 million primes calculated for every million separately. (b) The relation between the natural logarithm of the prime numbers and Brody parameter $\omega$ from one million up to 350 million primes calculated for every million separately.

Prime numbers are known to be distributed randomly among the integers, and their NNSD provides evidence that prime numbers are statistically random. The idea behind NNSD is to analyze how the gaps between adjacent numbers in a number sequence fluctuate around the average spacing [59]. For this, a well-defined average spacing is required. But the average spacing (averaged over many successive spacings, but not over the entire sequence) may not be uniform throughout the sequence. If this local average changes significantly on the scale of a single spacing, then there is no hope of separating the large-scale variation in the spacings from the local fluctuations. However, if the local average varies only on scales that are large compared to a single spacing, then the effects of this variation can be separated from the local fluctuations using a process called unfolding. Therefore, the sequence of prime numbers must be unfolded before the NNSD for prime numbers are estimated $[27,56]$.

One way to avoid the problem of unfolding is by using the idea of the ratio of two consecutive level spacings which is independent of the local density of states, and it does not require unfolding [44, 65-69]. 


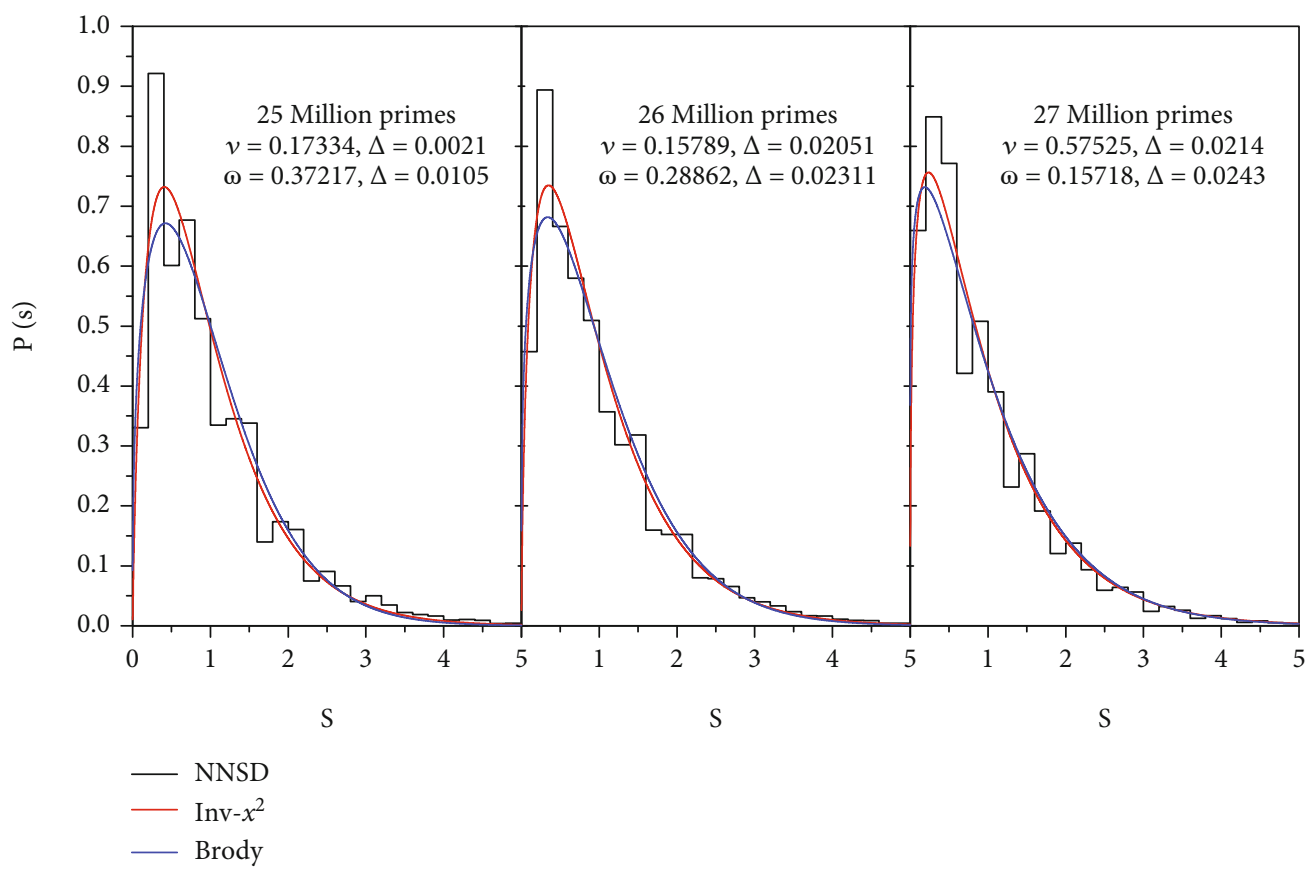

FIgURe 3: Nearest Neighbor Spacing Distribution (NNSD) for (a) the 25 million primes, (b) the 26 million primes, and (c) the 27 million primes with the best fit calculated using the inverse $\chi^{2}$ distribution Equation (3) and the Brody distribution Equation (7) including the average absolute deviation calculations $\Delta$.

As mentioned above, to use RMT in the analysis, the spacing between the consecutive prime numbers must be unfolded. Unfolding is a standard procedure for single long spectrum $[20,55,56]$. For this work, it is done using the unfolding formula stated in [21], given by Equations (5) and (6) as follows:

$$
\begin{aligned}
& d_{n}=p_{n+1}-p_{n}, \\
& D_{n}=\frac{d_{n}}{\operatorname{Ln}\left(p_{n}\right)+\left(d_{n} / p_{n}\right)} .
\end{aligned}
$$

Here, $d_{n}$ is the difference between any two successive primes and $D_{n}$ is the unfolded difference between any two successive primes; even for very large prime number $P_{n}$, we used the same equations, although in [21] the author ignored the term for large primes. Because the average distance between primes $\left(p_{n}-p_{n-1}\right)$ is $\operatorname{Ln}\left(p_{n}\right)$, we have from Equation (6) the unfolded spacings which have a mean value equal to unity. The unfolded specimen is then arranged and treated using the inverse $\chi^{2}$ distribution (3) and the Brody distribution (Equation (7)) [33-36, 39, 40, 50, 60].

The resulting NNSD of the mixed system, given by Equation (3), is used to study the statistical properties of the prime numbers where the prime numbers can be treated as energy levels of one-dimensional quantum systems [21, $28]$. As discussed in $[20,36,56,70,71]$, the NNSD of random numbers on a line can be given by the Poisson distribution (Equation (1)), whereas the NNSD of random numbers on a plane can be given by the Wigner surmise for Gaussian Orthogonal Ensemble (GOE) in Equation (2). Exploiting
TABLE 1: The fit parameters and absolute average deviation for the $25^{\text {th }}$ to $27^{\text {th }}$ million prime while the last three rows for $10^{4}$ primes around from $2.561 \times 10^{7}$ to $2.563 \times 10^{7}$.

\begin{tabular}{lcccc}
\hline$P_{n}$ & $v$ & $\Delta$ & $\omega$ & $\Delta$ \\
\hline $2.50 \times 10^{7}$ & 0.1733 & 0.0021 & 0.3722 & 0.0105 \\
$2.60 \times 10^{7}$ & -0.1579 & 0.0205 & 0.2886 & 0.0231 \\
$2.70 \times 10^{7}$ & -0.5753 & 0.0214 & 0.1572 & 0.0243 \\
$2.561 \times 10^{7}$ & 0.2021 & 0.0332 & 0.3777 & 0.0375 \\
$2.562 \times 10^{7}$ & -0.2567 & 0.0199 & 0.2591 & 0.0248 \\
$2.563 \times 10^{7}$ & -0.5610 & 0.0233 & 0.1601 & 0.0265 \\
\hline
\end{tabular}

this, there arises the possibility of describing the NNSD statistics of mixed systems as they exhibit transition between the Poisson and Wigner statistics as the underlying dynamic change occurs. The intermediate statistics can be described successfully by the Brody distribution. In this study, for comparison, we have used this popular distribution in the description of the mixed systems [10, 40-44]:

$$
P_{\text {Brody }}(\omega, s)=(\omega+1) a_{\omega} s^{\omega} e^{-a_{\omega} \omega^{\omega+1}}, \quad a_{\omega}=\Gamma^{\omega+1}\left(\frac{\omega+2}{\omega+1}\right) .
$$

The Brody distribution yields the Wigner-Dyson distribution if $\omega=1$, which indicates a fully chaotic case and leads to the Poisson distribution if $\omega=0$, for an integrable system. If $\omega$ takes any value between these two limiting values, we 


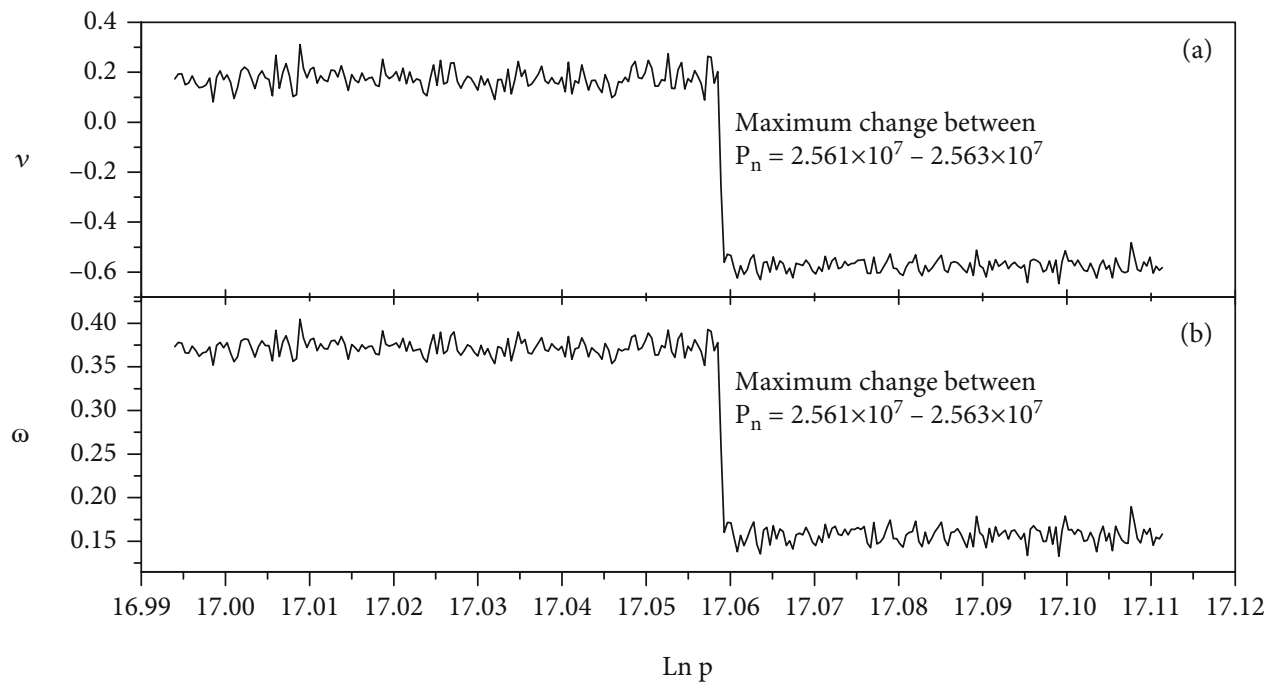

FIGURE 4: (a) The relation between the prime number of every $10^{4}$ primes separately and the inverse $\chi^{2}$ parameter $v$ from 24 million up to 27 million primes. (b) The relation between the prime number and Brody parameter $\omega$ for the same region and separation step.

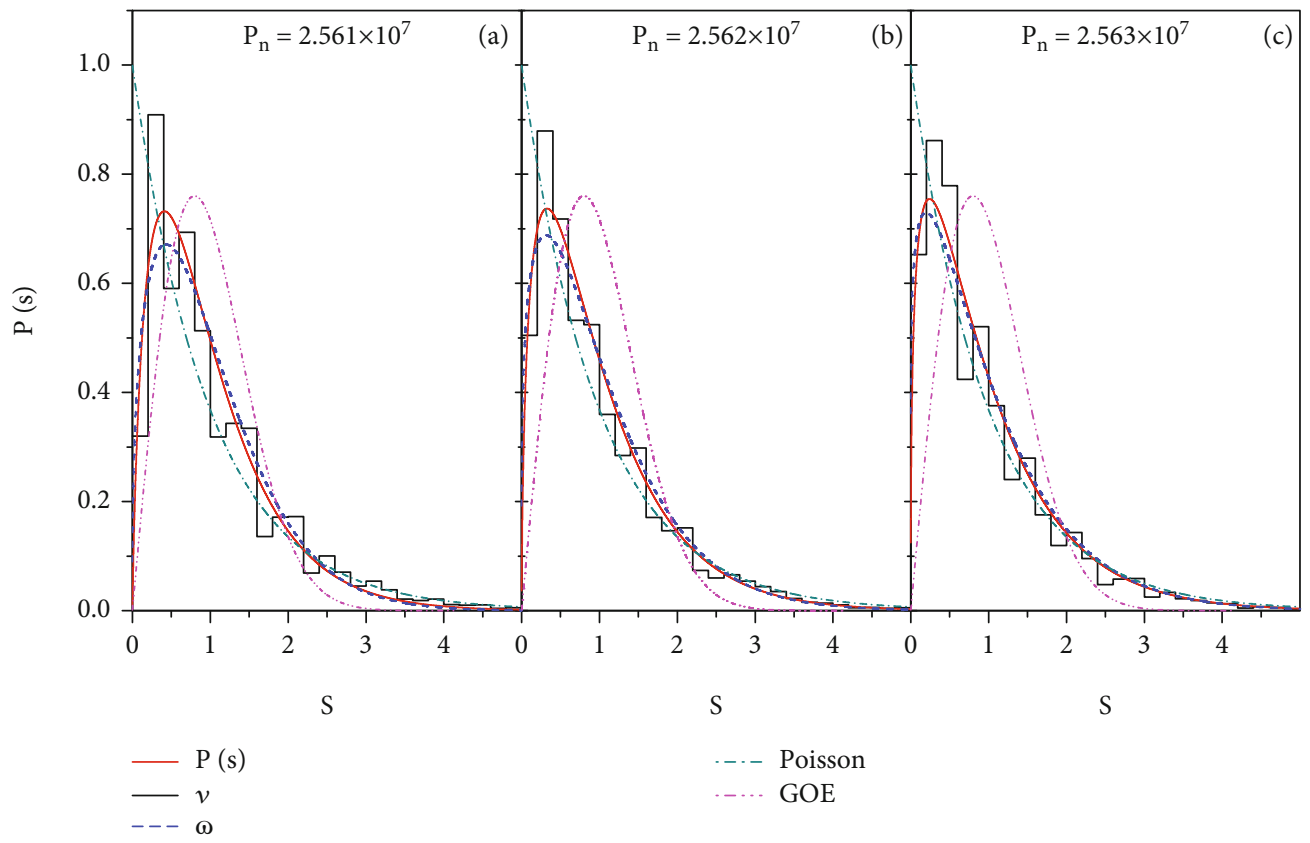

FIGURE 5: Nearest Neighbor Spacing Distribution (NNSD) for $10^{4}$ primes at (a) 25.61 million, (b) 25.62 million, and (c) 25.63 million, with the best fit calculated using the inverse $\chi^{2}$ distribution Equation (3) and the Brody distribution Equation (7). For reason of comparison the Poisson and GOE curves are also included.

say that the system is mixed and the chaoticity of the system increases as $\omega$ increases from 0 to $1[40]$.

\section{Results and Discussion}

This section discusses the analysis and the results of the proposed study. We have considered the sequences of the prime numbers as eigenvalues of a quantum physical system, and we used the NNSD of mixed systems to investigate the statistical properties of the spacing between adjacent prime numbers. The inverse $\chi^{2}$ distribution and the Brody distribution are considered for analysis.

We conducted the analysis for subsequences of every million prime separately, starting from the $1^{\text {st }}$ million to three hundred and fifty million primes. Figure 1 shows the relation between the prime numbers and the chaoticity parameters $v$ and $\omega$. We notice that at small values starting from the $1^{\text {st }}$ million, the behavior is hardly following the Poisson statistics. As we go further, the chaoticity of every individual million prime increases until the $25^{\text {th }}$ million, where it reaches maximum; then a sudden enhancement in 
TABLE 2: The calculated parameters for the inverse $\chi^{2}$ distribution $(v)$ and Brody distribution $(\omega)$ starting from 100 to 1.3 million primes.

\begin{tabular}{|c|c|c|c|c|c|c|c|c|}
\hline Prime no. & $v$ & $\omega$ & Prime no. & $v$ & $\omega$ & Prime no. & $v$ & $\omega$ \\
\hline $1.00 \times 10^{2}$ & 204.95 & 0.9899 & $8.00 \times 10^{3}$ & 0.3412 & 0.4086 & $2.00 \times 10^{5}$ & 0.2845 & 0.3921 \\
\hline $2.00 \times 10^{2}$ & 22.8189 & 0.9267 & $9.00 \times 10^{3}$ & 0.2633 & 0.3944 & $2.10 \times 10^{5}$ & 0.2970 & 0.3945 \\
\hline $3.00 \times 10^{2}$ & 3.9174 & 0.7850 & $1.00 \times 10^{4}$ & 0.1943 & 0.3817 & $2.20 \times 10^{5}$ & 0.3046 & 0.3960 \\
\hline $4.00 \times 10^{2}$ & 2.4857 & 0.7171 & $1.10 \times 10^{4}$ & 0.1565 & 0.3751 & $2.26 \times 10^{5}$ & 0.3076 & 0.3965 \\
\hline $5.00 \times 10^{2}$ & 2.0600 & 0.6783 & $1.20 \times 10^{4}$ & 0.1386 & 0.3724 & $2.27 \times 10^{5}$ & 0.3078 & 0.3965 \\
\hline $6.00 \times 10^{2}$ & 1.9258 & 0.6623 & $1.30 \times 10^{4}$ & 0.1182 & 0.3686 & $2.28 \times 10^{5}$ & 0.3091 & 0.3965 \\
\hline $7.00 \times 10^{2}$ & 1.8687 & 0.6515 & $1.40 \times 10^{4}$ & 0.1059 & 0.3669 & $2.29 \times 10^{5}$ & 0.3106 & 0.3970 \\
\hline $8.00 \times 10^{2}$ & 1.9348 & 0.6553 & $1.50 \times 10^{4}$ & 0.0884 & 0.3641 & $2.30 \times 10^{5}$ & 0.3104 & 0.3970 \\
\hline $9.00 \times 10^{2}$ & 2.0103 & 0.6622 & $1.60 \times 10^{4}$ & 0.1005 & 0.3667 & $2.31 \times 10^{5}$ & 0.3101 & 0.3969 \\
\hline $1.00 \times 10^{3}$ & 2.1030 & 0.6706 & $1.70 \times 10^{4}$ & 0.0753 & 0.3612 & $2.32 \times 10^{5}$ & 0.3101 & 0.3970 \\
\hline $1.10 \times 10^{3}$ & 2.2225 & 0.6822 & $1.80 \times 10^{4}$ & 0.0789 & 0.3615 & $2.33 \times 10^{5}$ & 0.3107 & 0.3971 \\
\hline $1.20 \times 10^{3}$ & 2.2450 & 0.6850 & $1.85 \times 10^{4}$ & 0.0762 & 0.3612 & $2.34 \times 10^{5}$ & 0.3131 & 0.3975 \\
\hline $1.30 \times 10^{3}$ & 2.2664 & 0.6872 & $1.88 \times 10^{4}$ & 0.0728 & 0.3605 & $2.35 \times 10^{5}$ & 0.3127 & 0.3975 \\
\hline $1.40 \times 10^{3}$ & 2.3222 & 0.6909 & $1.89 \times 10^{4}$ & 0.0721 & 0.3603 & $2.36 \times 10^{5}$ & 0.3085 & 0.3967 \\
\hline $1.50 \times 10^{3}$ & 2.3555 & 0.6937 & $1.90 \times 10^{4}$ & 0.0712 & 0.3602 & $2.37 \times 10^{5}$ & 0.3045 & 0.3960 \\
\hline $1.60 \times 10^{3}$ & 2.5208 & 0.7074 & $1.92 \times 10^{4}$ & 0.0823 & 0.3626 & $2.38 \times 10^{5}$ & 0.2995 & 0.3951 \\
\hline $1.70 \times 10^{3}$ & 2.4542 & 0.7011 & $2.00 \times 10^{4}$ & 0.0837 & 0.3628 & $2.39 \times 10^{5}$ & 0.2957 & 0.3945 \\
\hline $1.80 \times 10^{3}$ & 2.5981 & 0.7136 & $3.00 \times 10^{4}$ & 0.0906 & 0.3620 & $2.40 \times 10^{5}$ & 0.2931 & 0.3940 \\
\hline $1.90 \times 10^{3}$ & 2.5815 & 0.7120 & $4.00 \times 10^{4}$ & 0.1372 & 0.3706 & $2.50 \times 10^{5}$ & 0.2547 & 0.3872 \\
\hline $2.00 \times 10^{3}$ & 2.5828 & 0.7107 & $5.00 \times 10^{4}$ & 0.1562 & 0.3738 & $2.60 \times 10^{5}$ & 0.2193 & 0.3805 \\
\hline $2.10 \times 10^{3}$ & 2.5046 & 0.7046 & $6.00 \times 10^{4}$ & 0.1661 & 0.3738 & $2.70 \times 10^{5}$ & 0.1874 & 0.3742 \\
\hline $2.20 \times 10^{3}$ & 2.5471 & 0.7075 & $7.00 \times 10^{4}$ & 0.1732 & 0.3736 & $2.80 \times 10^{5}$ & 0.1593 & 0.3684 \\
\hline $2.30 \times 10^{3}$ & 2.6387 & 0.7151 & $8.00 \times 10^{4}$ & 0.1757 & 0.3736 & $2.90 \times 10^{5}$ & 0.1375 & 0.3639 \\
\hline $2.40 \times 10^{3}$ & 2.5775 & 0.7099 & $9.00 \times 10^{4}$ & 0.1716 & 0.3725 & $3.00 \times 10^{5}$ & 0.1179 & 0.3596 \\
\hline $2.50 \times 10^{3}$ & 2.4920 & 0.7023 & $1.00 \times 10^{5}$ & 0.1831 & 0.3739 & $4.00 \times 10^{5}$ & 0.0029 & 0.3325 \\
\hline $2.60 \times 10^{3}$ & 2.3515 & 0.6863 & $1.10 \times 10^{5}$ & 0.1941 & 0.3758 & $5.00 \times 10^{5}$ & -0.0485 & 0.3195 \\
\hline $2.70 \times 10^{3}$ & 2.1900 & 0.6699 & $1.20 \times 10^{5}$ & 0.1996 & 0.3761 & $6.00 \times 10^{5}$ & -0.0777 & 0.3120 \\
\hline $2.80 \times 10^{3}$ & 2.11638 & 0.6613 & $1.30 \times 10^{5}$ & 0.2045 & 0.3767 & $7.00 \times 10^{5}$ & -0.0903 & 0.3084 \\
\hline $2.90 \times 10^{3}$ & 2.0298 & 0.6515 & $1.40 \times 10^{5}$ & 0.2171 & 0.3793 & $8.00 \times 10^{5}$ & -0.0964 & 0.3066 \\
\hline $3.00 \times 10^{3}$ & 1.9260 & 0.6389 & $1.50 \times 10^{5}$ & 0.2339 & 0.3827 & $9.00 \times 10^{5}$ & -0.0996 & 0.3059 \\
\hline $4.00 \times 10^{3}$ & 1.1293 & 0.5348 & $1.60 \times 10^{5}$ & 0.2454 & 0.3848 & $1.00 \times 10^{6}$ & -0.1033 & 0.3050 \\
\hline $5.00 \times 10^{3}$ & 0.8284 & 0.4898 & $1.70 \times 10^{5}$ & 0.2581 & 0.3872 & $1.10 \times 10^{6}$ & -0.1054 & 0.3047 \\
\hline $6.00 \times 10^{3}$ & 0.5543 & 0.4454 & $1.80 \times 10^{5}$ & 0.2712 & 0.3898 & $1.20 \times 10^{6}$ & -0.1107 & 0.3024 \\
\hline $7.00 \times 10^{3}$ & 0.4416 & 0.4260 & $1.90 \times 10^{5}$ & 0.2816 & 0.3917 & $1.30 \times 10^{6}$ & -0.1148 & 0.3005 \\
\hline
\end{tabular}

regularity is reached in the $26^{\text {th }}$ and $27^{\text {th }}$ million regions. The calculated parameters for the $27^{\text {th }}$ million primes are $v=-0.5753$ and $\omega=0.1572$ for the inverse $\chi^{2}$ distribution and Brody distribution which showed the most regular region of the entire tested data. The rest of the data shows the Poissonian-like behavior with tendency to increase the chaoticity very slowly as shown in Figure 1.

The same behavior is shown in Figure 2; as we plotted the same data with logarithmic prime scale, the graphs show sudden decrease in chaoticity parameters at the $25^{\text {th }}-27^{\text {th }}$ million region which may mean that the gaps between adjacent prime numbers in these regions become smaller for large number of primes. Furthermore, Figure 3 shows the NNSD for subsequence of unfolded primes for $25^{\text {th }}, 26^{\text {th }}$, and $27^{\text {th }}$ million regions together with the best fit using Equations (3) and (7). Figure 3 clearly shows the transition from nearly chaotic at $25^{\text {th }}$ million region to nearly regular $27^{\text {th }}$ million region. We quantify the quality of the fits by 
TABLE 3: The calculated parameters for the inverse $\chi^{2}$ distribution $(v)$ and Brody distribution $(\omega)$ starting from 1.4 million primes to 350 million primes (continued).

\begin{tabular}{|c|c|c|c|c|c|c|c|c|}
\hline Prime no. & $v$ & $\omega$ & Prime no. & $v$ & $\omega$ & Prime no. & $v$ & $\omega$ \\
\hline $1.40 \times 10^{6}$ & -0.1176 & 0.2991 & $3.50 \times 10^{7}$ & -0.1121 & 0.2982 & $1.85 \times 10^{8}$ & -0.4193 & 0.2164 \\
\hline $1.50 \times 10^{6}$ & -0.1206 & 0.2978 & $4.00 \times 10^{7}$ & -0.1741 & 0.2816 & $1.90 \times 10^{8}$ & -0.4193 & 0.2165 \\
\hline $1.60 \times 10^{6}$ & -0.1231 & 0.2966 & $4.50 \times 10^{7}$ & -0.2204 & 0.2689 & $1.95 \times 10^{8}$ & -0.4193 & 0.2166 \\
\hline $1.70 \times 10^{6}$ & -0.1242 & 0.2960 & $5.00 \times 10^{7}$ & -0.2558 & 0.2590 & $2.00 \times 10^{8}$ & -0.4193 & 0.2167 \\
\hline $1.80 \times 10^{6}$ & -0.1244 & 0.2957 & $5.50 \times 10^{7}$ & -0.2838 & 0.2512 & $2.05 \times 10^{8}$ & -0.4192 & 0.2169 \\
\hline $1.90 \times 10^{6}$ & -0.1233 & 0.2959 & $6.00 \times 10^{7}$ & -0.3063 & 0.2448 & $2.10 \times 10^{8}$ & -0.4190 & 0.2170 \\
\hline $2.00 \times 10^{6}$ & -0.1225 & 0.2960 & $6.50 \times 10^{7}$ & -0.3248 & 0.2396 & $2.15 \times 10^{8}$ & -0.4188 & 0.2172 \\
\hline $3.00 \times 10^{6}$ & -0.1096 & 0.2980 & $7.00 \times 10^{7}$ & -0.3398 & 0.2354 & $2.20 \times 10^{8}$ & -0.4185 & 0.2174 \\
\hline $4.00 \times 10^{6}$ & -0.0961 & 0.3005 & $7.50 \times 10^{7}$ & -0.3525 & 0.2319 & $2.25 \times 10^{8}$ & -0.4182 & 0.2176 \\
\hline $5.00 \times 10^{6}$ & -0.0779 & 0.3039 & $8.00 \times 10^{7}$ & -0.3631 & 0.2290 & $2.30 \times 10^{8}$ & -0.4180 & 0.2177 \\
\hline $6.00 \times 10^{6}$ & -0.0617 & 0.3074 & $8.50 \times 10^{7}$ & -0.3723 & 0.2265 & $2.35 \times 10^{8}$ & -0.4177 & 0.2179 \\
\hline $7.00 \times 10^{6}$ & -0.0465 & 0.3110 & $9.00 \times 10^{7}$ & -0.3800 & 0.2244 & $2.40 \times 10^{8}$ & -0.4174 & 0.2181 \\
\hline $8.00 \times 10^{6}$ & -0.0328 & 0.3143 & $9.50 \times 10^{7}$ & -0.3868 & 0.2226 & $2.45 \times 10^{8}$ & -0.4171 & 0.2183 \\
\hline $9.00 \times 10^{6}$ & -0.0182 & 0.3182 & $1.00 \times 10^{8}$ & -0.3924 & 0.2212 & $2.50 \times 10^{8}$ & -0.4168 & 0.2185 \\
\hline $1.00 \times 10^{7}$ & -0.0055 & 0.3217 & $1.05 \times 10^{8}$ & -0.3964 & 0.2203 & $2.55 \times 10^{8}$ & -0.4165 & 0.2187 \\
\hline $1.10 \times 10^{7}$ & 0.0058 & 0.3249 & $1.10 \times 10^{8}$ & -0.4000 & 0.2195 & $2.60 \times 10^{8}$ & -0.4162 & 0.2189 \\
\hline $1.20 \times 10^{7}$ & 0.0156 & 0.3277 & $1.15 \times 10^{8}$ & -0.4032 & 0.2187 & $2.65 \times 10^{8}$ & -0.4158 & 0.2191 \\
\hline $1.30 \times 10^{7}$ & 0.0247 & 0.3303 & $1.20 \times 10^{8}$ & -0.4062 & 0.2181 & $2.70 \times 10^{8}$ & -0.4155 & 0.2193 \\
\hline $1.40 \times 10^{7}$ & 0.0324 & 0.3326 & $1.25 \times 10^{8}$ & -0.4087 & 0.2176 & $2.75 \times 10^{8}$ & -0.4151 & 0.2195 \\
\hline $1.50 \times 10^{7}$ & 0.0397 & 0.3347 & $1.30 \times 10^{8}$ & -0.4105 & 0.2172 & $2.80 \times 10^{8}$ & -0.4146 & 0.2196 \\
\hline $1.60 \times 10^{7}$ & 0.0458 & 0.3364 & $1.35 \times 10^{8}$ & -0.4121 & 0.2169 & $2.85 \times 10^{8}$ & -0.4137 & 0.2198 \\
\hline $1.70 \times 10^{7}$ & 0.0516 & 0.3381 & $1.40 \times 10^{8}$ & -0.4136 & 0.2167 & $2.90 \times 10^{8}$ & -0.4130 & 0.2200 \\
\hline $1.80 \times 10^{7}$ & 0.0567 & 0.3396 & $1.45 \times 10^{8}$ & -0.4148 & 0.2165 & $2.95 \times 10^{8}$ & -0.4123 & 0.2201 \\
\hline $1.90 \times 10^{7}$ & 0.0618 & 0.3410 & $1.50 \times 10^{8}$ & -0.4160 & 0.2164 & $3.00 \times 10^{8}$ & -0.4116 & 0.2203 \\
\hline $2.00 \times 10^{7}$ & 0.0664 & 0.3424 & $1.55 \times 10^{8}$ & -0.4169 & 0.2163 & $3.05 \times 10^{8}$ & -0.4109 & 0.2204 \\
\hline $2.10 \times 10^{7}$ & 0.0706 & 0.3436 & $1.60 \times 10^{8}$ & -0.4177 & 0.2162 & $3.10 \times 10^{8}$ & -0.4102 & 0.2205 \\
\hline $2.20 \times 10^{7}$ & 0.0746 & 0.3447 & $1.65 \times 10^{8}$ & -0.4185 & 0.2162 & $3.15 \times 10^{8}$ & -0.4095 & 0.2207 \\
\hline $2.30 \times 10^{7}$ & 0.0784 & 0.3458 & $1.70 \times 10^{8}$ & -0.4192 & 0.2161 & $3.20 \times 10^{8}$ & -0.4089 & 0.2208 \\
\hline $2.40 \times 10^{7}$ & 0.0818 & 0.3468 & $1.71 \times 10^{8}$ & -0.4193 & 0.2161 & $3.25 \times 10^{8}$ & -0.4082 & 0.2210 \\
\hline $2.50 \times 10^{7}$ & 0.0852 & 0.3478 & $1.72 \times 10^{8}$ & -0.4193 & 0.2161 & $3.30 \times 10^{8}$ & -0.4076 & 0.2211 \\
\hline $2.60 \times 10^{7}$ & 0.0756 & 0.3456 & $1.73 \times 10^{8}$ & -0.4193 & 0.2162 & $3.35 \times 10^{8}$ & -0.4070 & 0.2213 \\
\hline $2.70 \times 10^{7}$ & 0.0471 & 0.3387 & $1.74 \times 10^{8}$ & -0.4193 & 0.2162 & $3.40 \times 10^{8}$ & -0.4064 & 0.2214 \\
\hline $2.80 \times 10^{7}$ & 0.0212 & 0.3323 & $1.75 \times 10^{8}$ & -0.4193 & 0.2162 & $3.45 \times 10^{8}$ & -0.4057 & 0.2216 \\
\hline $2.90 \times 10^{7}$ & -0.0026 & 0.3263 & $1.76 \times 10^{8}$ & -0.4193 & 0.2162 & $3.50 \times 10^{8}$ & -0.4051 & 0.2217 \\
\hline $3.00 \times 10^{7}$ & -0.0246 & 0.3208 & $1.80 \times 10^{8}$ & -0.4194 & 0.2163 & & & \\
\hline
\end{tabular}

their absolute average deviation:

$$
\Delta=\frac{1}{N_{L}} \sum\left|P_{\text {cal }}-P_{\text {Fit }}\right| \text {, }
$$

where $P_{\text {cal }}, P_{\text {Fit }}$, and $N_{L}$ are the calculated NNSD, the best-fit using Equations (3) and (7), and the number of values measured, respectively. The best-fit values of the parameters together with their absolute average deviation $\Delta$ are given in Table 1. 


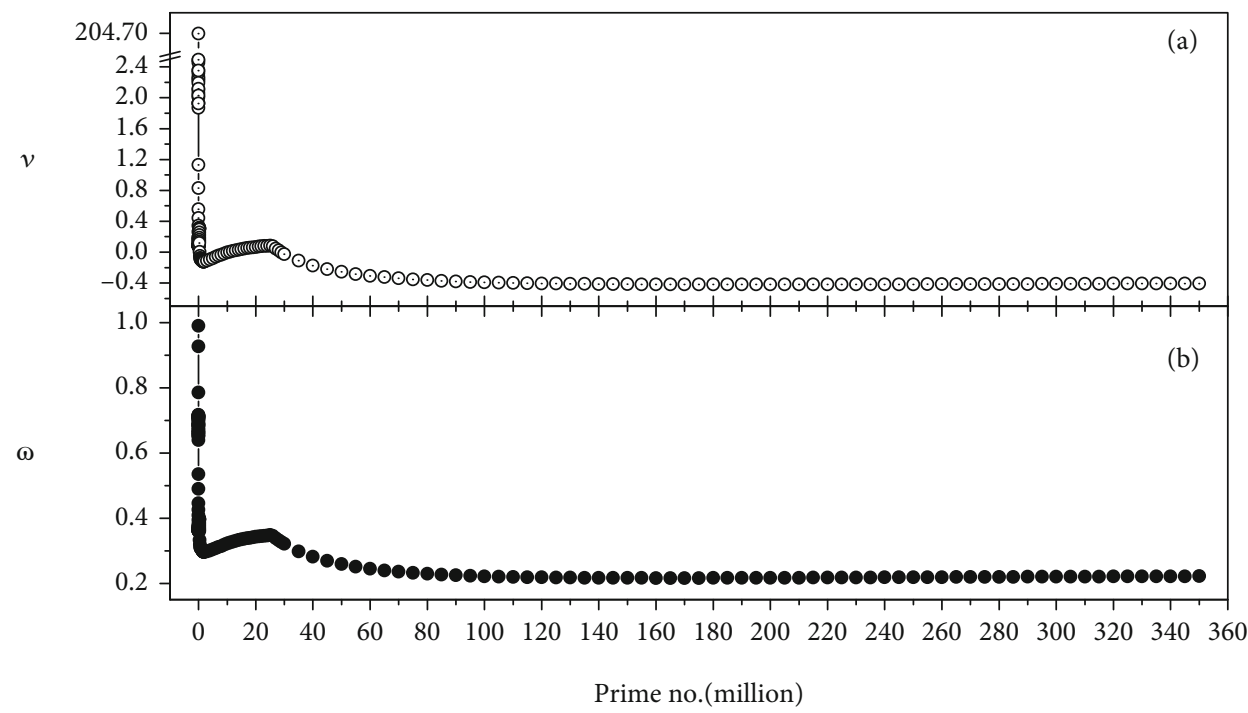

Figure 6: (a) The relation between the accumulated prime numbers and the inverse $\chi^{2}$ parameter $v$ from 100 to 350 million primes. (b) The relation between the accumulated prime numbers and the Brody parameter $\omega$ from 100 to 350 million primes.

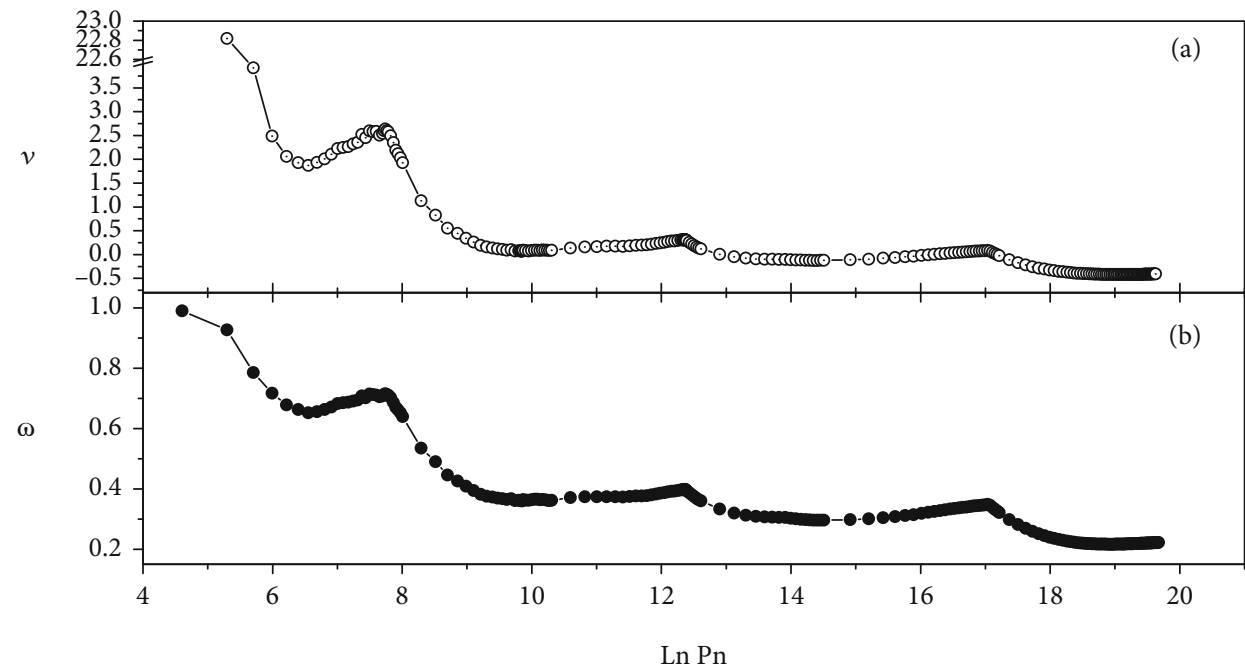

Figure 7: (a) The relation between the natural logarithm of the accumulated prime numbers from 200 to 350 million primes and the inverse $\chi^{2}$ parameter $v$. (b) The relation between the natural logarithm of the accumulated prime numbers from 100 to 350 million primes and the Brody parameter $\omega$.

To investigate precisely where there are sudden increases in the regularity in the $25^{\text {th }}-27^{\text {th }}$ million region, we conducted analysis and calculate the chaoticity parameters for smaller subsequences of every $10^{4}$ primes separately, starting from the $24^{\text {th }}$ million to $27^{\text {th }}$ million primes. Figure 4 shows the relation between prime numbers and the chaoticity parameters $v$ and $\omega$ using a logarithmic scale. We can clearly locate the region of sudden transfer between large chaoticity at $2.561 \times 10^{7}$ and high regularity at $2.563 \times 10^{7}$.

Figure 5 shows the NNSD histogram for subsequence of $10^{4}$ unfolded primes at (a) $2.561 \times 10^{7}$, at (b) $2.562 \times 10^{7}$, and at (c) $2.563 \times 10^{7}$ together with the best fit using Equations (3) and (7), and for the reason of comparison, we include the Poisson and GOE curves. Figure 5 clearly shows the transition from nearly chaotic at prime number $2.561 \times 10^{7}$ region to nearly regular at $2.563 \times 10^{7}$ region. Table 1 shows the values of the chaoticity parameters $v$ and $\omega$ and the absolute average deviation $\Delta$ between calculated NNSD and the fitted values. We can notice that the two distributions can fit the calculated NNSD, but inverse- $\chi^{2}$ superstatistics distribution gives better agreement with the investigated prime than the Brody distribution.

Next, the distribution was made up of sequences of the first $N$ prime numbers from one hundred to three hundred and fifty million prime numbers, where the size of the specimen is increased in steps. During the calculation of the parameters of the $1^{\text {st }}$ and $2^{\text {nd }}$ million, we take steps as low as 100 prime increment and as large as $10^{5}$ prime numbers. The choice was made according to the region of the prime numbers. It was taken around the minima and maxima (that 


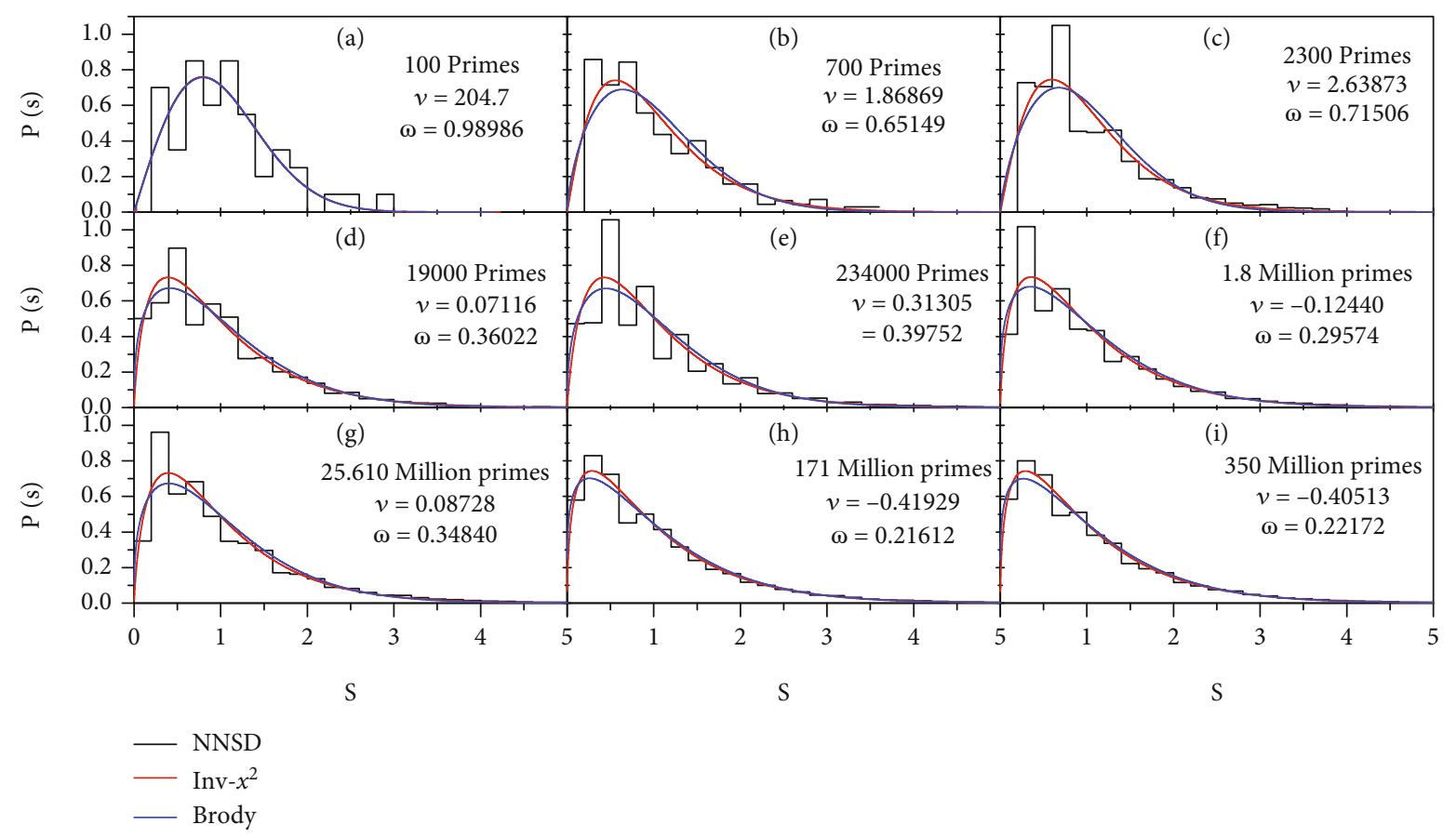

Figure 8: (NNSD) for continuous sequences of unfolded primes using (a) 100 primes, (b) 700 primes, (c) 2300 primes, (d) $1.90 \times 10^{4}$ primes, (e) $2.34 \times 10^{5}$ primes, (f) $1.80 \times 10^{6}$ primes, (g) $2.561 \times 10^{7}$ primes, (h) $1.71 \times 10^{8}$ primes, and $3.50 \times 10^{8}$ primes. The curves show the best fit calculated using the inverse $\chi^{2}$ distribution Equation (3) and the Brody distribution Equation (7).

appeared during the calculations) in smaller steps to determine it accurately.

Starting from the beginning of the $3^{\text {rd }}$ million of the prime numbers, the increments are taken to be one million due to the small change in the values of the calculated parameters of Equations (3) and (7). After thirty million prime numbers, the steps were made at every 5 million except at 170 million prime number regions as shown in Tables 2 and 3 and Figures 6 and 7. In all the sequences, we take the bin size of the calculated data to be 0.2 .

Our studies of the NNSD of the difference between consecutive primes showed that the system of the prime numbers becomes more regular as the number of the primes increases and hardly becomes out of chaos at 700 primes (first minima); at 100 primes, the system is fully chaotic and can be described by Equation (2); this can be represented graphically by Figure 8 (a) for 100 primes and Figure 6(b) for 700 primes. Further increase in the prime numbers made the system more chaotic and reaches the first maxima at prime number 2300 (see Figure 6(c)). This behavior repeated several times through the tested data as shown in Figure 7 and Tables 2 and 3. A number of successive minima and maxima were found during the calculations. Figures $8(\mathrm{~b})-8(\mathrm{~h})$ show histograms with best fit using Equations (3) and (7) for this successive minima and maxima; for the reason of comparison, we present the 100 primes and the 350 million primes in the same figure. Also, Table 4 shows the fit parameters $v$ and $\omega$ and absolute average deviation $\Delta$ for the same accumulated prime distributions in Figure 8.

We can notice that the system of prime numbers becomes more regular and approaches the Poissonian distri-
TABLE 4: The fit parameters and absolute average deviation for the accumulated prime sequences shown in Figure 8.

\begin{tabular}{lcccc}
\hline$P_{n}$ & $v$ & $\Delta$ & $\omega$ & $\Delta$ \\
\hline $10^{2}$ & 204.7 & 0.0592 & 0.9899 & 0.0592 \\
$7.00 \times 10^{2}$ & 1.8687 & 0.0385 & 0.6515 & 0.0452 \\
$2.30 \times 10^{3}$ & 2.6387 & 0.0410 & 0.7151 & 0.0489 \\
$1.90 \times 10^{4}$ & 0.07116 & 0.0255 & 0.3602 & 0.0258 \\
$2.34 \times 10^{5}$ & 0.3131 & 0.0454 & 0.3975 & 0.0441 \\
$1.8 \times 10^{6}$ & -0.1244 & 0.0279 & 0.2957 & 0.0314 \\
$2.561 \times 10^{7}$ & 0.0852 & 0.0266 & 0.3478 & 0.0311 \\
$1.71 \times 10^{8}$ & -0.4193 & 0.0131 & 0.2161 & 0.0180 \\
$3.50 \times 10^{8}$ & -0.4051 & 0.0120 & 0.2217 & 0.0163 \\
\hline
\end{tabular}

bution as the number of the primes largely increased. The relation between the logarithmic prime numbers and the inverse $\chi^{2}$ distribution (Figure $7(\mathrm{a})$ ) and the Brody distribution parameters (Figure $7(\mathrm{~b})$ ) shows this behavior of successive minima and maxima more clearly.

One of the most prominent features of this work is the big change in the values of the chaoticity permeates around the $25^{\text {th }}-27^{\text {th }}$ million region. In addition to the study of every separate $10^{4}$ primes at this region (see Figure 4 and Table 1), we conduct another analysis for accumulated size of the prime number from 24 million to 27 million primes with increasing steps ranging between $10^{4}$ around the peak at $2.561 \times 10^{7}$ to $10^{5}$ far from it. Figures 9 and 10 show this study with normal and logarithmic scales. We notice a 


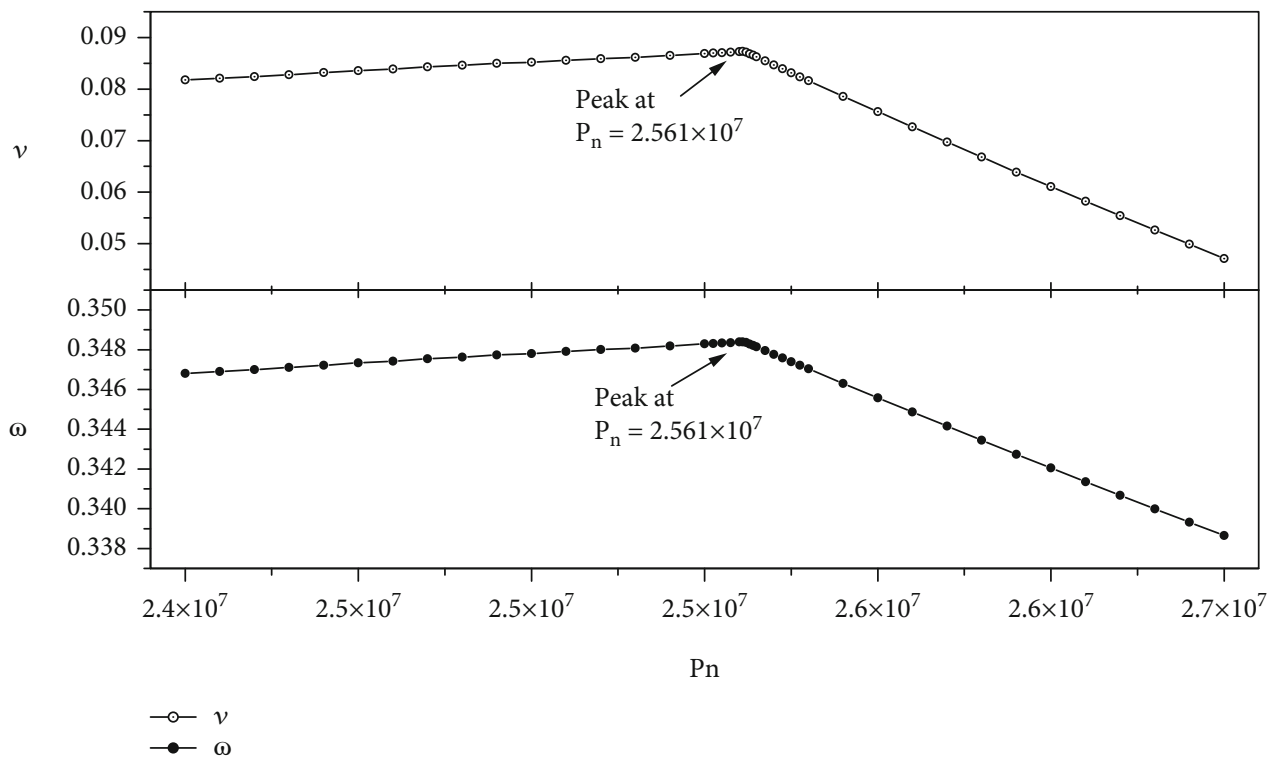

FIGURE 9: (a) The relation between the accumulated prime numbers and the inverse $\chi^{2}$ parameter $v$ from 24 million to 27 million primes with small steps. (b) The relation between the accumulated prime numbers and the Brody parameter $\omega$ from 24 million to 27 million primes with small steps.

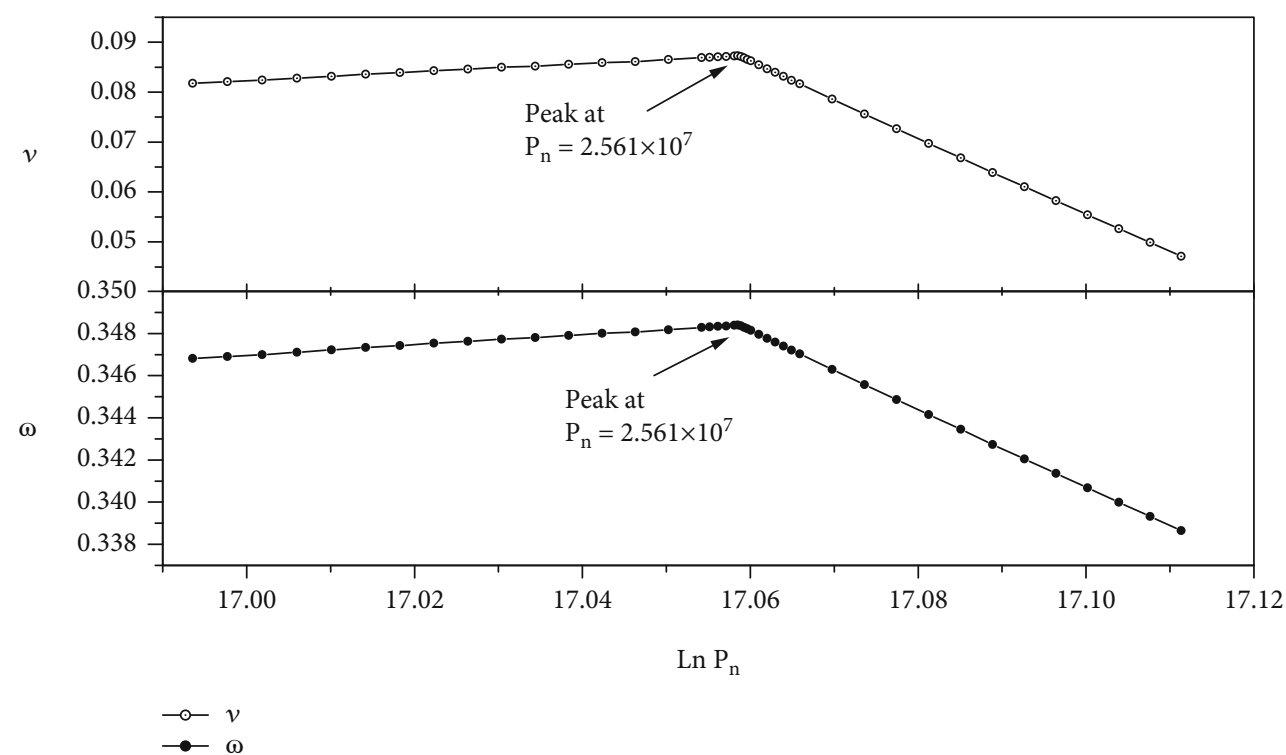

Figure 10: (a) The relation between the natural logarithm of the accumulated prime numbers and the inverse $\chi^{2}$ parameter $v$ from 24 million to 27 million primes with small steps. (b) The relation between the natural logarithm of the accumulated prime numbers and the Brody parameter $\omega$ from 24 million to 27 million primes with small steps.

smooth and slow change around the peak. The change in the fit parameters is very slow for large accumulated size of the prime numbers than for small size of the prime numbers.

The logarithmic period between two successive minima or maxima can be calculated using the logarithmic difference between any of them (except the $1^{\text {st }}$ minima), as shown in Table 5. This difference for minima is $\Delta \operatorname{Ln}\left(P_{n \text { min }}\right)=4.553$ on average (for the last two periods). This enables us to predict the region of the next minima. We can roughly calculate it as $\operatorname{Ln}\left(P_{n \min }\right)=23.513 \longrightarrow P_{n \min }=1.6277 \times 10^{10}$. Also, the logarithmic difference between two successive maxima $\Delta \operatorname{Ln}\left(P_{n \max }\right)=4.659$ on average. Hence, the next maxima can roughly be calculated as $\operatorname{Ln}\left(P_{n \text { max }}\right)=21.717$ which yields $P_{n \text { max }}=2.7013 \times 10^{9}$.

An alternative way for predicting the next minima and maxima by plotting the values of minima and maxima with respect to its number $(m)$ and linearly fit them as shown in Figure 11, the $1^{\text {st }}$ minima is omitted in the lower curve 11-b. The linear fitting equations are given by Equation (9) for maxima and Equation (10) for minima. 


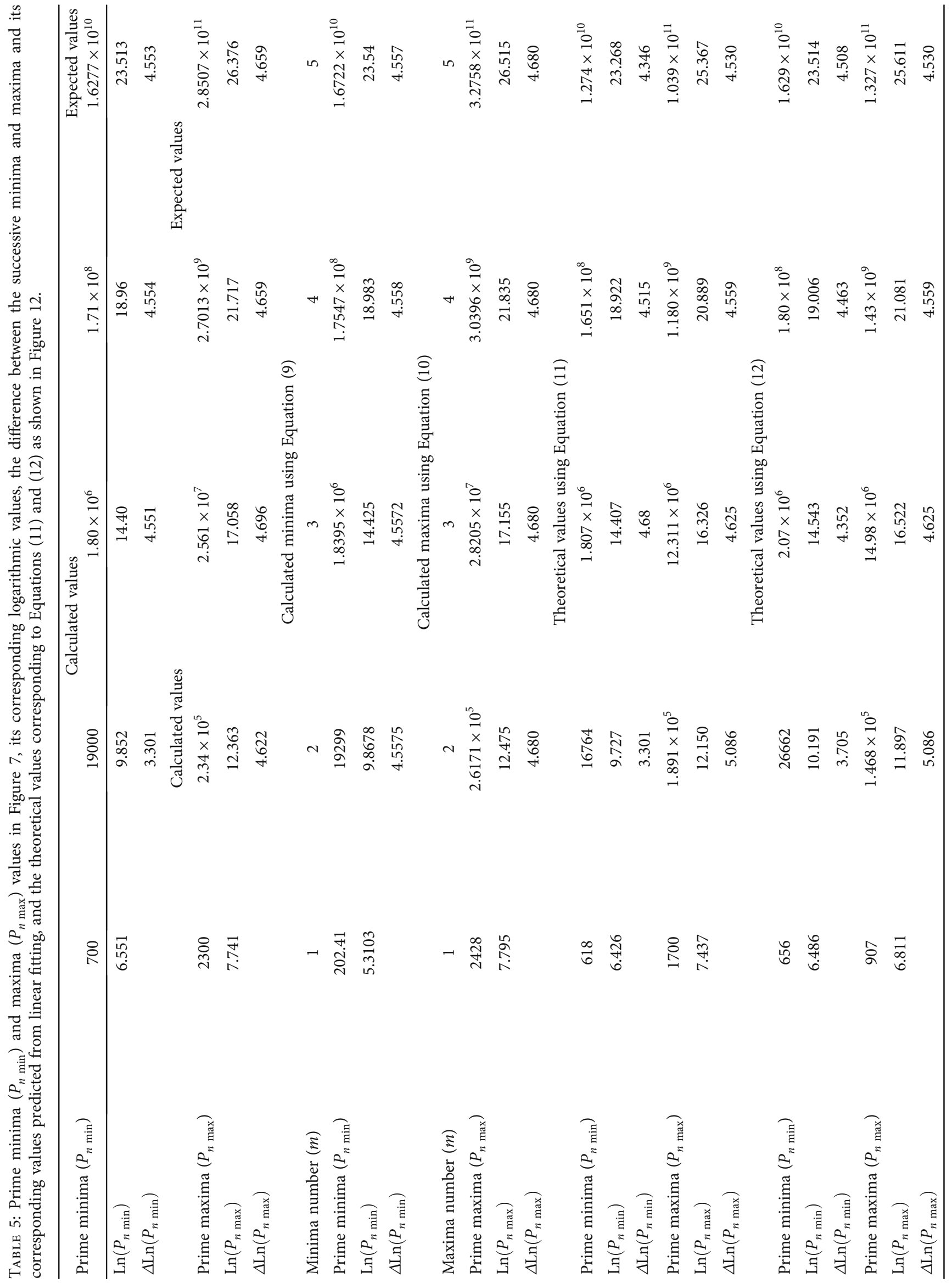




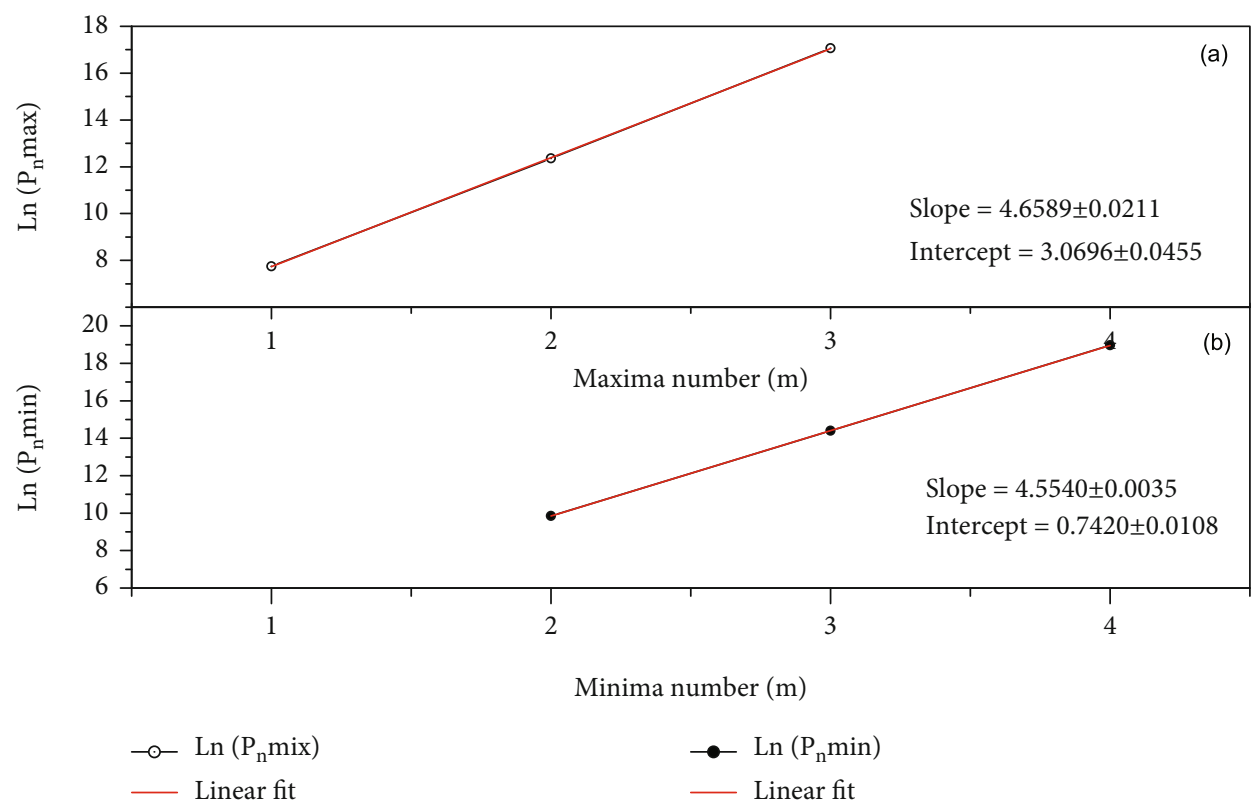

FIGURE 11: (a) The relation between the maxima, its maxima number $(m)$, and its corresponding liner fit. (b) The relation between the minima and its minima number and its corresponding liner fit.

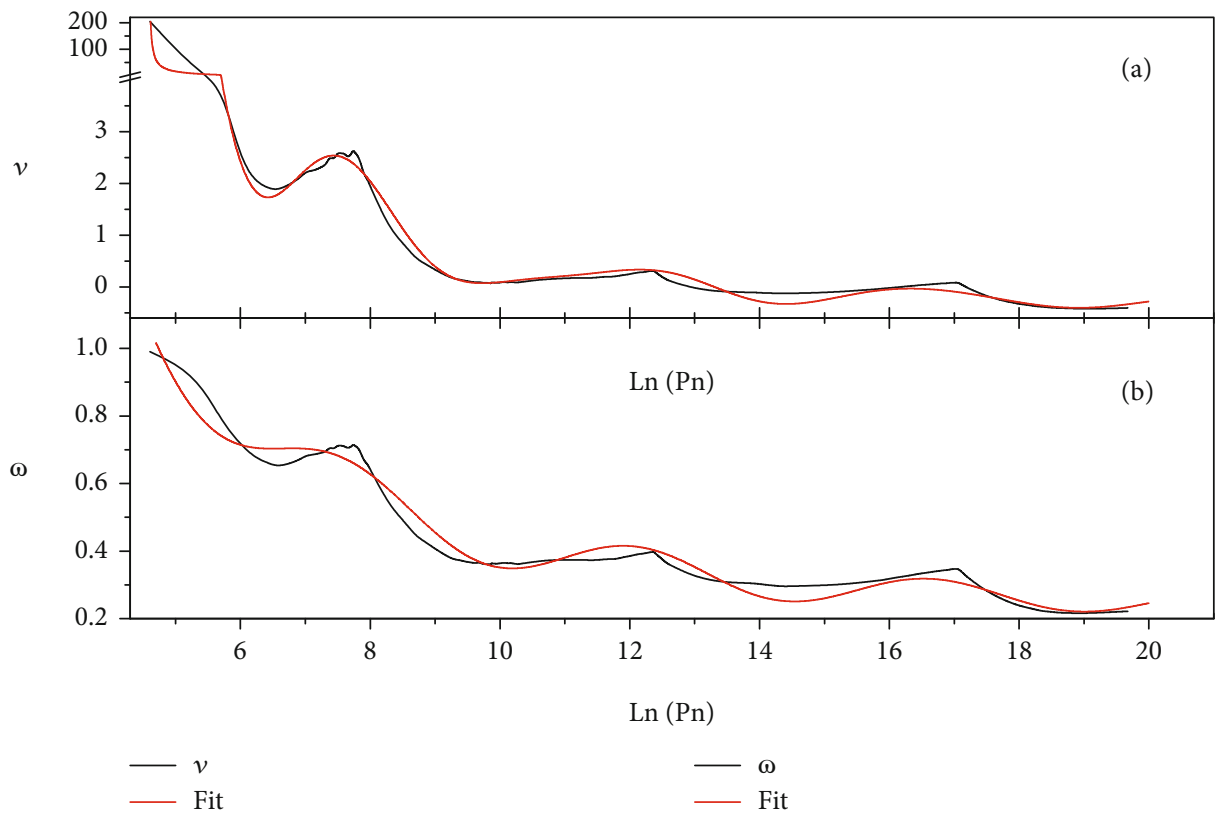

FIGURE 12: (a) The relation between the natural logarithm of the accumulated prime numbers from 100 to 350 million primes and the inverse $\chi^{2}$ parameter $v$ and the fitted values using Equation (11). (b) The relation between the natural logarithm of the accumulated prime numbers from 100 to 350 million primes and the Brody parameter $\omega$ and the fitted values using Equation (12).

$L_{n} P_{n} \min (m)=(4.5540 \pm 0.0035) m+(0.7420 \pm 0.0108)$

$L_{n} P_{n} \max (m)=(4.6589 \pm 0.0211) m+(3.0696 \pm 0.0455)$.

We can use the last two equations to calculate all the minima and maxima (see Table 5). The $5^{\text {th }}$ minima can be cal- culated by putting $m=5$ in Equation (9): $\operatorname{Ln} P_{n \min }(5)=23.54$ which yields $P_{n \text { min }}(5)=1.6722 \times 10^{10}$. The $4^{\text {th }}$ maxima can be calculated by putting $m=4$ in Equation (10); so $\operatorname{Ln} P_{n \text { max }}(4)$ $=21.835$ which yields $P_{n \max }(4)=3.0396 \times 10^{9}$.

Hence, we can expect the $4^{\text {th }}$ maxima in the prime range between $2.7024 \times 10^{9}$ and $3.0396 \times 10^{9}$ and the $5^{\text {th }}$ minima in the prime range between $1.6277 \times 10^{10}$ and $1.6722 \times 10^{10}$. This will be the subject of future work to calculate as many primes as to locate the next minima and maxima. 
In an attempt to find an empirical equation for the results in Tables 2 and 3 and Figure 7, we proposed the following formula:

$$
\begin{gathered}
v=\frac{12.8687 e^{-0.220169 x} \cos ^{2}(1.20823 x)-2.45332 e^{-0.0127408 x} \sin (1.41977 x)+e^{-0.215933 x}+4.70948}{x-4.58008}-0.6056 \\
\omega=\frac{-45.7898 e^{-0.0656143 x}+43.767 e^{-0.0843228 x} \sin ^{2}(0.691538 x)+44.0825 e^{-0.0880813 x} \cos ^{2}(0.695294 x)+9.50654}{x+0.115366}
\end{gathered}
$$

In the two pervious formulas, we consider $x=\operatorname{Ln} P_{n}$. The fitted values using the two equations compared to the calculated values of $v$ and $\omega$ are plotted in Figure 12(a) for $v$ and Figure 12(b) for $\omega$. The two equations showed accepted behavior and reproduced most of the minima and maxima fairly. We can notice that the fitted curve in Figure 12(a) using Equation (11) gives better agreement with the calculated data than that of the fitted curve in Figure 12(b) using Equation (12).

In Table 5, we present a comparison between the calculated and theoretical values of prime numbers minima and maxima. The theoretical values are in good agreement with the calculated values especially at the logarithmic scale.

In Equation (11), as $x=\operatorname{Ln} P_{n} \longrightarrow \infty, v=-0.6056$, while for Equation (12) as $x=\operatorname{Ln} P_{n} \longrightarrow \infty, \omega=0$.

This may verify our assumption of the Poissonian behavior of the prime numbers at very large values.

\section{Conclusion}

We have considered the sequences of prime numbers as eigenvalues of a quantum physical system, and we used the NNSD of mixed systems to investigate the statistical properties of the spacing between adjacent prime numbers. We used the inverse $\chi^{2}$ distribution and the Brody distribution, which are powerful tools for investigating the regularchaos mixed systems. We found that the system of prime numbers is a mixed system that tends to become a Poissonian as the prime numbers become very large.

This conclusion may be considered a first step, and it will be further examined in a future work using an extensive and detailed study for a wider range of prime numbers using the same method and other methods like the idea of the ratio of two consecutive level spacings. Also the longer-range statistics, i.e., the spectral rigidity and level number variance, will be one of the major metrics.

\section{Data Availability}

Data are available on request (please contact M. AbdelMageed at m.abdulasim@qu.edu.sa).

\section{Conflicts of Interest}

The authors declare that they have no conflicts of interest.

\section{Acknowledgments}

The authors gratefully acknowledge Qassim University, represented by the Deanship of Scientific Research, for the financial support of this research under the number cosao-bs-2019-2-2I-5712 during the academic year 1440 AH/2019 AD.

\section{References}

[1] A. Salim, W. Osamy, A. M. Khedr, A. Aziz, and M. AbdelMageed, "A secure data gathering scheme based on properties of primes and compressive sensing for IoT-based WSNs," IEEE Sensors Journal, vol. 21, no. 4, pp. 5553-5571, 2021.

[2] A. M. Khedr and D. M. Omar, "ERPLBC: energy efficient routing protocol for load balanced clustering in wireless sensor networks," Ad Hoc \& Sensor Wireless Networks, vol. 42, pp. 145-169, 2018.

[3] A. Aziz, K. Singh, W. Osamy, and A. M. Khedr, "Effective algorithm for optimizing compressive sensing in IoT and periodic monitoring applications," Journal of Network and Computer Applications, vol. 126, no. 15, pp. 12-28, 2019.

[4] W. Osamy, A. Salim, and A. M. Khedr, "An information entropy based-clustering algorithm for heterogeneous wireless sensor networks," Wireless Networks, vol. 26, no. 3, pp. 18691886, 2020.

[5] W. Osamy, A. M. Khedr, A. Aziz, and A. El-Sawy, "Clustertree routing scheme for sata gathering in periodic monitoring applications," IEEE Access, vol. 6, pp. 77372-77387, 2019.

[6] W. Osamy, A. M. Khedr, A. A. El-Sawy, A. Salim, and D. Vijayan, "IPDCA: intelligent proficient data collection approach for IoT-enabled wireless sensor networks in smart environments," Electronics, vol. 10, no. 9, p. 997, 2021.

[7] A. M. Khedr, "Effective data acquisition protocol for multihop heterogeneous wireless sensor networks using compressive sensing," Algorithms, vol. 8, no. 4, pp. 910-928, 2015.

[8] A. M. Odlyzko, "On the distribution of spacings between zeros of the zeta function," Mathematics of Computation, vol. 48, no. 177 , p. $273,1987$.

[9] B. Cipra, "Prime formula weds number theory and quantum physics," Science, vol. 274, no. 5295, pp. 2014-2015, 1996.

[10] G. Maino, "Prime numbers, atomic nuclei, symmetries and superconductivity," AIP Conference Proceedings, vol. 2150, article 030009, 2019.

[11] F. W. K. Firk and S. J. Miller, "Nuclei, primes and the random matrix connection," Symmetry, vol. 1, no. 1, pp. 64-105, 2009. 
[12] J. Garcia-Sandoval, "Fractals and discrete dynamics associated to prime numbers," Solitons \& Fractals, vol. 139, article 110029, 2020.

[13] J. Sakhr, R. K. Bhaduri, and B. P. van Zyl, "Zeta function zeros, powers of primes, and quantum chaos," Physical Review E, vol. 68 , no. 2, article 026206, 2003.

[14] M. V. Berry, "Quantum chaology, prime numbers and Riemann's zeta function," Institute of Physics Conference Series, vol. 133, 1993.

[15] M. Stallings, Cryptography and Network Security: Principles and Practice, Prentice-Hall, New Jersey, 1999.

[16] E. Goles, O. Schulz, and M. Markus, "Prime number selection of cycles in a predator-prey model," Complexity, vol. 6 , no. 4 , 38 pages, 2001.

[17] J. Toha and M. A. Soto, "Biochemical identification of prime numbers," Medical Hypotheses, vol. 53, no. 4, p. 361, 1999.

[18] E. D. Shalit, "Prime numbers-why are they so exciting?," Frontiers for Young Minds, vol. 6, 2018.

[19] A. Fraile, R. Martinez, and D. Fernandez, "Jacob's ladder: prime numbers in 2D," Mathematical and Computational Applications, vol. 25, no. 1, p. 5, 2020.

[20] M. L. Mehta, Random Matrices, Academic Press, Boston, 2nd edition, 1991.

[21] M. Wolf, "Nearest-neighbor-spacing distribution of prime numbers and quantum chaos," Physical Review E, vol. 89, no. 2, article 022922, 2014.

[22] C. E. Porter, "Statistical theories of spectra," in Fluctuations, Academic Press, 1965.

[23] J. Robert, L. Oliver, and K. Soundararajan, "Unexpected biases in the distribution of consecutive primes," Proceedings of the National Academy of Sciences of the United States of America, vol. 113, no. 31, pp. E4446-E4454, 2016.

[24] M. Gardner, "Mathematical games," Scientific American, vol. 210, no. 1, pp. 120-128, 1964.

[25] Z. Gamba, J. Hernando, and L. Romanelli, “Are prime numbers regularly ordered?," Physics Letters A, vol. 145, no. 2-3, pp. 106-108, 1990.

[26] M. R. Schroeder, Number Theory in Science and Communications, Springer Series in Information Sciences, Springer, Berlin, 1986.

[27] T. Timberlake, "Random numbers and random matrices: quantum chaos meets number theory," American Journal of Physics, vol. 74, no. 6, pp. 547-553, 2006.

[28] T. K. Timberlake and J. M. Tucker, "Is there quantum chaos in the prime numbers?," https://arxiv.org/abs/.0708.2567v2.

[29] R. L. Liboff and M. Wong, "Quasi-chaotic property of the prime-number sequence," International Journal of Theoretical Physics, vol. 37, no. 12, pp. 3109-3117, 1998.

[30] M. Wolf, "Applications of statistical mechanics in number theory,” Physica A, vol. 274, no. 1-2, pp. 149-157, 1999.

[31] M. Wolf, " $1 / f$ noise in the distribution of prime numbers," Physica A, vol. 241, no. 3-4, pp. 493-499, 1997.

[32] P. J. Richens and M. V. Berry, "Pseudointegrable systems in classical and quantum mechanics," Physica D: Nonlinear Phenomena, vol. 2, no. 495, 1981.

[33] A. Y. Abul-Magd, "Random matrix theory within superstatistics," Physical Review E, vol. 72, no. 6, article 066114, 2005.

[34] A. Y. Abul-Magd, "Superstatistics in random matrix theory," SQU Journal for Science, vol. 17, no. 2, pp. 157-169, 2017.
[35] A. Y. Abul-Magd, "Nonextensive random-matrix theory based on Kaniadakis entropy," Physics Letters A, vol. 361, no. 6, pp. 450-454, 2007.

[36] A. Y. Abul-Magd, B. Dietz, T. Friedrich, and A. Richter, "Spectral fluctuations of billiards with mixed dynamics: from time series to superstatistics," Physical Review E, vol. 77, no. 4, article 046202, 2008.

[37] C. Beck and E. G. D. Cohen, "Superstatistics," Physica A, vol. 322, pp. 267-275, 2003.

[38] C. Beck, "Superstatistics in hydrodynamic turbulence," Physica D: Nonlinear Phenomena, vol. 193, no. 1-4, pp. 195-207, 2004.

[39] M. Abdel-Mageed and A. Y. Abul-Magd, "Analysis of symmetry breaking in quartz blocks using superstatistical randommatrix theory," Physica A, vol. 391, no. 11, pp. 3027-3032, 2012.

[40] T. Brody, "A statistical measure for the repulsion of energy levels," Identifying migration from political data, Viana do Castelo, Minho, Portugal, 1827-1895, vol. 7, no. 12, pp. 482484, 1973.

[41] C. P. Dettmann, O. Georgiou, and G. Knight, "Spectral statistics of random geometric graphs," EPL (Europhysics Letters), vol. 118, no. 1, article 18003, 2017.

[42] M. Matsuo, T. Døssing, E. Vigezzi, and S. Aberg, "Level statistics of near-yrast states in rapidly rotating nuclei," Nuclear Physics A, vol. 620, no. 3, pp. 296-316, 1997.

[43] L. Munoz, R. A. Molina, and J. M. G. Gömez, "Chaos in nuclei: theory and experiment," Journal of Physics: Conference Series, vol. 1023, no. 1, article 012011, 2018.

[44] N. D. Chavda, H. N. Deota, and V. K. B. Kota, "Poisson to GOE transition in the distribution of the ratio of consecutive level spacings," Physics Letters A, vol. 378, no. 41, pp. 30123017, 2014.

[45] K. Cho and T. Miyano, "Chaotic cryptography using augmented Lorenz equations aided by quantum key distribution," IEEE Transactions on Circuits and Systems I: Regular Papers, vol. 62, no. 2, article 478487, 2015.

[46] Y. Zhang, "Bounded gaps between primes," Annals of Mathematics, vol. 179, no. 3, pp. 1121-1174, 2014.

[47] M. Wolf, "Multifractality of prime numbers," Physica A, vol. 160, no. 1, pp. 24-42, 1989.

[48] K. Ford, B. Green, S. Konyagin, and T. Tao, "Large gaps between consecutive prime numbers," Annals of Mathematics, vol. 183, no. 3, pp. 935-974, 2016.

[49] A. Kourbatov and M. Wolf, "Predicting maximal gaps in sets of primes," Mathematics, vol. 7, no. 5, p. 400, 2019.

[50] A. Y. Abul-Magd, "Superstatistics in random matrix theory," Physica A, vol. 361, no. 1, pp. 41-54, 2006.

[51] A. Y. Abul-Magd and M. H. Simbel, "Phenomenological model for symmetry breaking in a chaotic system," Physical Review E, vol. 70, no. 4, article 046218, 2004.

[52] M. V. Berry and M. Tabor, "Level clustering in the regular spectrum," Proceedings of the Royal Society of London. Series A, vol. 356, pp. 375-394, 1977.

[53] O. Bohigas, M. J. Giannoni, and C. Schmit, "Characterization of chaotic quantum spectra and universality of level fluctuation Laws," Physical Review Letters, vol. 52, no. 1, pp. 1-4, 1984.

[54] O. Bohigas, Chaos and Quantum Physics, M.-J. Giannoni, A. Voros, J. Zinn-Justin, and L. Houches, Eds., Summer School, Session LII, North-Holland, Amsterdam, 1991. 
[55] T. Guhr, A. Muller-Groeling, and H. A. Weidenmüller, "Random-matrix theories in quantum physics: common concepts," Physics Reports, vol. 299, no. 4-6, pp. 189-425, 1998.

[56] O. Evnin and W. Piensuk, "Quantum resonant systems, integrable and chaotic," Journal of Physics A, vol. 52, no. 2, article 025102, 2019.

[57] A. J. Lichtenberg and M. A. Lieberman, "Regular and stochastic motion," in Applied Mathematical Sciences, Springer, New York, 1983.

[58] C. Chandre and H. R. Jauslin, "Renormalization-group analysis for the transition to chaos in Hamiltonian systems," Physics Reports, vol. 365, no. 1, pp. 1-64, 2002.

[59] J. Zakrzewski, K. Dupret, and D. Delande, "Statistical properties of energy levels of chaotic systems: Wigner or non-Wigner?," Physical Review Letters, vol. 74, no. 4, pp. 522-525, 1995.

[60] M. V. Berry and M. Robnik, "Semiclassical level spacings when regular and chaotic orbits coexist," Journal of Physics A: Mathematical and General, vol. 17, no. 12, pp. 2413-2421, 1984.

[61] C. Beck, E. G. D. Cohen, and H. L. Swinney, "From time series to superstatistics," Physical Review E, vol. 72, no. 5, article 056133, 2005.

[62] J. X. de Carvalho, M. S. Hussein, M. P. Pato, and A. J. Sargeant, "Perturbative treatment of symmetry breaking within random matrix theory," Physics Letters A, vol. 372, p. 4898, 2008.

[63] I. S. Gradshteyn and I. M. Ryzhik, Tables of Integrals, Series, and Products, Academic, New York, 1980.

[64] E. Bogomolny, U. Gerald, and C. Schmit, "Models of intermediate spectral statistics," Physical Review E, vol. 59, no. 2, pp. R1315-R1318, 1999.

[65] V. Oganesyan and D. A. Huse, "Localization of interacting fermions at high temperature," Physical Review B, vol. 75, no. 15, article 155111, 2007.

[66] Y. Y. Atas, E. Bogomolny, O. Giraud, and G. Roux, "distribution of the ratio of consecutive level spacings in random matrix ensembles," Physical Review Letters, vol. 110, no. 8, article 084101, 2013.

[67] Y. Y. Atas, E. Bogomolny, O. Giraud, P. Vivo, and E. Vivo, "Joint probability densities of level spacing ratios in random matrices," Journal of Physics A: Mathematical and Theorertical, vol. 46, no. 35, article 355204, 2013.

[68] U. T. Bhosale, S. H. Tekur, and M. S. Santhanam, "Scaling in the eigenvalue fluctuations of correlation matrices," Physical Review E, vol. 98, no. 5, article 052133, 2018.

[69] A. Sarkar, M. Kothiyal, and S. Kumar, "Distribution of the ratio of two consecutive level spacings in orthogonal to unitary crossover ensembles," Physical Review E, vol. 101, no. 1, article 012216, 2020

[70] J. Sakhr and J. M. Nieminen, "Poisson-to-Wigner crossover transition in the nearest-neighbor statistics of random points on fractals," Physical Review E, vol. 72, article 045204R, 2005.

[71] F. Haake, Quantum Signatures of Chaos, Springer, Berlin, 2nd edition, 2001. 